\title{
There and Back to the Present: An Evolutionary Tale on Biological
}

3 Leandro Duarte ${ }^{1 *}$, Gabriel Nakamura ${ }^{1}$, Vanderlei Debastiani ${ }^{1}$, Renan Maestri ${ }^{1}$, Maria

4 João Ramos Pereira ${ }^{1}$, Marcus Cianciaruso ${ }^{2}$, José Alexandre F. Diniz-Filho ${ }^{2}$

$5{ }^{1}$ Programa de Pós-graduação em Ecologia, Instituto de Biociências, Universidade

6 Federal do Rio Grande do Sul

7 Av. Bento Gonçalves 9500 CP 15007 Porto Alegre, 91501-970, Brazil

$8 \quad{ }^{2}$ Programa de Pós-graduação em Ecologia e Evolução, Instituto de Ciências

9 Biológicas, Universidade Federal de Goiás. CP 131, 74690-900, Goiânia, GO, Brazil

$10 *$ Corresponding author: duarte.1das@gmail.com

11 Running head: Niche evolution predicts diversity

12 Keywords: Ornstein-Uhlenbeck model, adaptive rate, Approximate Bayesian

13 Computation, eco-evolutionary dynamics

14 ABSTRACT: Ecologists often agree on the importance of macroevolution for nichemediated distribution of biological diversity along environmental gradients. Yet, macroevolutionary diversification and dispersal in time and space generate uneven geographic distribution of phylogenetic pools, which affects the imprint let by macroevolution on local species pools. In this article we introduce an individual-based simulation approach coupled to Approximate Bayesian Computation (ABC) that allows to parameterize the adaptation rate of species' niche positions along the evolution of a monophyletic lineage, and the intensity of dispersal limitation, associated with the distribution of biological diversity between assemblages potentially connected by dispersal (metacommunity). The analytical tool was implemented in an R package called $m c f l y$. We evaluated the statistical performance of the analytical framework using simulated datasets, which confirmed the suitability of the analysis to estimate adaptation rate and dispersal limitation parameters. Further, we evaluated the role played by niche evolution and dispersal limitation on species diversity distribution of Phyllostomidae bats across the Neotropics. The framework proposed here shed light on the links between niche evolution, dispersal limitation and the distribution of biological diversity, and thereby improved our understanding of evolutionary imprints on ecological 
bioRxiv preprint doi: https://doi.org/10.1101/2021.12.11.472171; this version posted December $14,2021$. The copyright holder for this

preprint (which was not certified by peer review) is the author/funder, who has granted bioRxiv a license to display the preprint in perpetuity. It is made available under aCC-BY-NC-ND 4.0 International license.

31 patterns. Perhaps more importantly, it offers new possibilities for solving the eco-

32 evolutionary puzzle. 


\section{Introduction}

34 Why some places have so many species while others have so few? This is a central question that generations of ecologists after G. E. Hutchinson have been trying to answer after his seminal article (Hutchinson 1959). Indeed, biological diversity is the most widely addressed feature of ecological assemblages; yet, its interpretation may be tricky (Ricotta 2005). The mechanisms determining the striking variation of biological diversity over space and across environmental gradients have been debated for decades (Vellend 2016). Alternative (and complementary) theories have been proposed to explain biological diversity, either based on species niches (Hutchinson 1957; Levine and HilleRisLambers 2009), neutral processes (Hubbell 2001; MacArthur and Levins 1967) or biogeographic and historical factors (Gerhold et al. 2018; MacArthur 1972; Ricklefs and Schluter 1993).

Over the last decades, community phylogenetics (Cavender-Bares et al. 2009; Mouquet et al. 2012; Webb et al. 2002) has posited that phylogenetic relationships among sympatric species may link niche evolution to biogeography and, ultimately, help understanding patterns in regional and local biological diversity patterns. Accordingly, niche divergence between closely related species may be somehow prevented along evolutionary history (Felsenstein 1985; Harvey and Pagel 1991; Nosil 2012). Such tendency of species niche to vary less than expected by chance along evolution is often interpreted as evidence of phylogenetic niche conservatism (Losos 2008; Pyron et al. 2015; Wiens and Graham 2005). If so, and whether dispersal plays only a minor role in species assembly process (which is very disputable, see Vellend 2016), phylogenetic niche conservatism would provide an explicit causal link between the phylogenetic diversity of local communities and major ecological mechanisms (environmental filtering, biotic interactions, see Webb et al. 2002). Following this rationale, some ecologists have been advocating the use of phylogenetic covariation as a proxy for niche similarity among species (Cadotte et al. 2012; Tucker et al. 2018).

Nonetheless, evidence against such 'phylogeny-as-proxy' approach has been accumulated over the last years, as similar phylogenetic diversity patterns may be explained by different, and often opposing, mechanisms (Gerhold et al. 2015; Godoy et al. 2014; Mayfield and Levine 2010; Swenson 2019). As controversial conclusions on the usefulness of interpreting phylogenetic diversity in the light of ecological mechanisms promoting biological diversity proliferated, further theoretical advances in community phylogenetics have decelerated over the last years (but see Cadotte et al. 
2017; Tucker et al. 2018). Tracing back plausible evolutionary scenarios underlying species distribution patterns across current environmental gradients remains a main challenge for community phylogenetics (Webb et al. 2002).

Unravelling evolutionary processes underlying niche divergence might help explaining the distribution of extant species belonging to a given clade across ecological gradients (McPeek 2017; Weber et al. 2017). Nonetheless, such enterprise faces two big challenges. First, theoretical and empirical evidence often demonstrate that the inference of eco-evolutionary niche dynamics based on static estimates of phylogenetic signal in species traits is not unequivocal (Münkemüller et al. 2015; Revell et al. 2008). This is because strong phylogenetic signal in species traits can emerge from processes other than niche conservatism, including neutral evolution (Cooper et al. 2010; Gould and Lewontin 1979; Harvey and Pagel 1991; Nosil 2012). Indeed, under high adaptive rate pulling species niches to an optimum value (which might be also interpreted as niche conservatism), phylogenetic signal in the niche is completely vanished (Butler and King 2004; Hansen et al. 2008). Second, ecologists often measure species traits to characterize their niches (Elton 1927; McPeek 2017), under the sound premise that species niches are mostly molded by ecological pressures determining phenotypic patterns of species (Ackerly 2003; Hansen and Martins 1996). In this sense, trait dominance and/or complementarity across environmental gradients (Garnier et al. 2016) is often taken as evidence of niche-mediated species assembly patterns. However, species traits correlate among themselves due to their shared evolutionary history, which results in complex trait-environment relationships. Therefore, inferring which specific trait(s) explain the distribution of biological diversity across environmental gradients is not trivial (Duarte et al. 2018). Indeed, searching for an evolutionary basis of niche-based species assembly across environmental gradients is still a topic of debate among ecologists (Cadotte et al. 2017; McPeek 2017; Tucker et al. 2018; Weber et al. 2017).

If neither phylogenetic signal in niche traits nor trait-environment correlations allow to build a solid bridge between niche evolution and species assembly across environmental gradients, then what? A starting point is to consider under which circumstances we should expect a detectable imprint of evolutionary processes on biological diversity. First, such imprint only makes sense if we consider a monophyletic lineage with most branches well represented across the geographic space under analysis. Otherwise, we may be investigating phylogenetic signal in biological diversity based on 
101 species (1) whose evolution was independent for the most time lineages have been

102 diversifying, for example, tree ferns and angiosperms (Duarte et al. 2012) or Anura and

103 Gymnophiona (Loyola et al. 2013), or (2) whose phylogenetic relationships are too

104 incomplete or fragmented to provide a realistic evolutionary scenario (Crisp and Cook

105 2005). However, even when the species pool belongs to a monophyletic, well

106 represented lineage, endemic to the region or biome we are interested in, the imprint left

107 by phylogeny on regional or local diversity patterns may either persist or be completely

108 erased (Maestri and Duarte 2020). This is because species assembly, and therefore

109 biological diversity, is an instantaneous snapshot of complex eco-evolutionary

110 dynamics acting upon co-occurring species (Shipley 2010; Weber et al. 2017). If the

111 adaptation rate of species from an ancestral niche position towards an optimum niche

112 condition, mediated by short-term biotic interactions (Paine et al. 2018) and/or

113 environmental filters (Kraft et al. 2015), was sufficiently strong to break phylogenetic

114 signal in species niches (Hansen et al. 2008), no phylogenetic imprint on species

115 assembly would be detected. Thus, it is not surprising that tracing back evolutionary

116 processes underlying biological diversity patterns based on static phylogenetic signal

117 among species may lead to disparate conclusions (Gerhold et al. 2015). Appropriate

118 tools for the evaluation of the influence of phylogeny on biological diversity across

119 space are necessary for this purpose.

120 A possible solution to solve this puzzle implies building alternative scenarios of

121 niche evolution given a phylogenetic hypothesis to generate expected species assembly

122 patterns. This should allow us to infer the most likely scenarios of niche evolution

123 determining empirical species assembly patterns based on the set of expectations that

124 most resemble the empirical data. In this article we introduce a model-based simulation

125 approach that allows to estimate the strength of adaptation rate along the niche

126 evolution of species belonging to a monophyletic lineage and distributed across

127 metacommunities, defined as sets of local assemblages connected by dispersal (Leibold

128 et al. 2004). Moreover, the new framework also allows to estimate to what extent

129 dispersal limitation influences diversity distribution across space. We start by defining

130 the theoretical aspects of niche- and neutral-based species assembly into local

131 assemblages, which provide the necessary background to a further contextualization of

132 niche evolution as a component of niche-based species assembly. Afterwards, we

133 introduce a rejection-based Approximate Bayesian Computation (ABC) algorithm

134 (Beaumont 2010; Csilléry et al. 2010) to estimate the strength of adaptation rate and 
135 dispersal limitation given a set of sites described by species belonging to a

136 monophyletic clade, a phylogenetic hypothesis for these species, spatial coordinates of

137 sites, and an environmental factor associated with species assembly patterns. We

138 demonstrate the usefulness of the simulation approach for inferring the imprint left by

139 niche evolution and dispersal limitation on species diversity patterns across

140 metacommunity and biogeographical scales by evaluating the statistical performance of

141 the ABC algorithm using simulated datasets. Further, we investigate the role played by

142 niche evolution and dispersal limitation on species diversity distribution of

143 Phyllostomidae bats across the Neotropics.

Parameterizing niche and neutral processes determining species assembly

145 Niche theory partially explains in part the distribution of biological diversity across

146 space. The availability of key resources and the prevalence of specific levels of

147 environmental conditions in a site enable populations of some species to thrive, while

148 others decrease their numbers for the same reason. Together with environmental

149 filtering, biotic interactions modulate the probability of maintenance of viable

150 populations of some species in the presence of others (Ackerly 2003; Hutchinson 1957;

151 MacArthur and Levins 1967; Vellend 2016; Violle et al. 2012, but see Kraft et al.

152 2015).

153 On the other hand, neutral dynamics relies on dispersal-based species assembly

154 to explain biological diversity. Neutral dynamics is modeled based on dispersal

155 limitation, immigration rates, local and regional species abundances, and speciation

156 events determining demography in local species assemblages, assuming that species are

157 ecologically equivalent in respect to their niches (Hubbell 2001). Even though no one

158 dispute the importance of niche-based processes to explain species assembly patterns,

159 dispersal processes also play an important role to determine species distribution across

160 space (Vellend 2016). Therefore, the importance of neutral processes (Bell 2000;

161 Hubbell 2001) cannot be underestimated. In fact, such dichotomy between niche and

162 neutral processes is only apparent (Gravel et al. 2006), since these processes represent

163 complementary mechanisms determining biological diversity (Vellend 2016). Both

164 niche and neutral processes provide a solid theoretical background to model species

165 assembly into local assemblages (Gravel et al. 2006) distributed in a metacommunity

166 context. 

some degree of connection by dispersal. Let $N_{*}$ be the number of individuals inhabiting each $k$ assemblage, and $N_{*_{M}}$ be the total number of individuals in the metacommunity, such that $N *_{M}=\sum_{k=1}^{M} N_{*_{k}}$. In the absence of a major disturbance, $N *_{k}$ is expected to increase until $k$ achieves carrying capacity $\left(J_{k}\right)$, quantified as the maximum number of individuals allowed to inhabit $k$, given the minimum amount of space and resources per capita demanded by the organisms (Del Monte-Luna et al. 2004). Thus, under $J_{k}$, we expect $d N_{* k}(t)=0$, and species assembly likely starts to follow a zero-sum dynamic (Hubbell 2001). This means that an individual currently inhabiting $k$ must necessarily die before a new one can be recruited, either by birth or immigration from other assemblage.

Given there is vacant space in $k$ to be occupied by new recruits, what processes will determine their identity? As we seen before, niche processes may be the answer to this question, at least partially. For a given species $i$ belonging to the species pool $q$, the niche position $y_{i}$ can be defined as the environmental condition that maximizes the probability of $N_{i k}>0$ such that $d N_{i k}(t) \geq 0$. Further, the niche breadth $s_{i}$ can be defined as the amount of deviation from $y_{i}$ that allows the conditions $N_{i k}>0$ and $d N_{i k}(t) \geq 0$ to be satisfied (Sexton et al. 2017). From a niche perspective, and considering only the influence of environmental filtering, the probability $\left(\lambda_{i k}\right)$ of species $i$ to recruit a new individual in $k$ can be modeled as a deterministic Gaussian function based on their niche position and breadth, as follows:

Accordingly, $x_{k}$ is the environmental position of $k$ across an environmental gradient, $y_{i}$ is the niche position of $i$ (measured in the same scale of $x_{k}$ ), and $s_{i}$ is the niche breadth of $i$. This model has been originally proposed by Minchin (1987), and has been adopted in several studies to simulate species assemblages (Dray and Legendre 2008; Duarte et al. 2018; Nakamura et al. 2020; Peres-Neto et al. 2017; Peres-Neto et al. 2012; Sokol et al. 2017; Sokol et al. 2015). incorporates the effect of biotic interactions (Gravel et al. 2006):

$$
R_{\text {Niche }_{i k}}=\frac{\lambda_{i k}}{\sum_{j \neq i}^{q} \lambda_{j k}} \text {. }
$$



$\in q$, which are also candidates to recruit new individuals, competing directly with $i$. Since $R_{\text {Niche }_{i k}}$ is only defined by niche position and breadth, $q$ can contain not only the species already resident in $k$, but also potential immigrant species occurring at any assemblage $l$, such that $l \in M$. The only condition to be a candidate recruiter species to $k$ is to show $R_{\text {Niche }_{i k}}>0$. This condition agrees with the species sorting model of metacommunity theory (Leibold et al. 2004). incorporating parameters from Hubbell's neutral model (Hubbell 2001), which provides spatial context to species assembly in local assemblages. First, let us consider dispersal limitation as a mechanism affecting the success of any species $i$ belonging to the regional species pool $q$, and occurring in any $l$ assemblage, to disperse new recruits to assemblage $k$. The probability $\delta_{l k}$ of any $i$ inhabiting $l$ to contribute to the immigrant pool of $k$ may be modeled as a Gaussian function of the shortest linear distance $(r$,

212 rescaled between 0 and 1, see Sokol et al. 2017) between $k$ and $l$, and the slope $w$ of the

213 dispersal kernel (the probability density function of the dispersal success from a source

214 to a sink assemblage, see Nathan and Muller-Landau 2000) for the species in $l$ (Gravel 215 et al. 2006; Sokol et al. 2017; Sokol et al. 2015), as follows:

$$
\delta_{l k}=e^{-w r_{l k}^{2}}
$$

217 For a slope $w=0, \delta_{l k}=1$, independently of the distance between $l$ and $k$, which means

218 that the species in assemblage $l$ face no barrier to disperse to $k$ (the assemblages are 219 panmictic). On the other hand, for slope $w=10, \delta_{l k}=0.9$ for sites showing $r=0.1$, but 220 decreases fast to $\delta_{l k}=0.08$ if the distance between the assemblages increases to $r=0.5$.

221 Over the next sections we introduce a simulation-based framework to estimate $w$ for 222 empirical datasets, which enables quantifying dispersal limitation underlying diversity 223 patterns (see Estimating niche and neutral parameters underlying species diversity gradients using Approximate Bayesian Computation).

The probability of any species $i$ to successfully disperse new recruits from any assemblage $l\left(R_{\text {Dispersal }_{i M}}\right)$, is a function of the abundance of $i$ across $M$, or $N_{i M}=$

$227 \sum_{l \neq k}^{M} N_{i l}$, and $\delta_{l k}$, and is contingent on the probability of dispersal for all other species $q$ in $M$ (Gravel et al. 2006), as follows: 


$$
R_{\text {Dispersal }_{i M}}=\frac{\sum_{l \neq k}^{M} N_{i l} \delta_{l k}}{\sum_{j \neq i}^{q} \sum_{l \neq k}^{M} N_{j l} \delta_{l k}} .
$$

230 It is important to observe that even for $\delta_{l k}=1$, immigrant species arriving to site $k$ may

231 not succeed as new colonizers, depending on how resistant $k$ is to immigration.

232 Assemblages where resident species are strong recruiters may prevent the entrance of

233 new colonizers (e.g. Duarte et al. 2006), which reduces the chance of new species

234 become part of the assemblage, even in the absence of dispersal limitation. For a site $k$

235 under zero-sum dynamic, let $d_{\mathrm{k}}$ be the number of instantaneous deaths in $k$. Site $k$ is

236 now open to the recruitment of $J_{k}-d_{k}$ new individuals, which may be generated either

237 by resident parents inhabiting $k\left(b_{k}\right)$, or by individuals dispersing from any site $l \in M$

$238\left(b_{l}\right)$, such that $J_{k}=\left(b_{k}-d_{k}\right)+b_{l}$. The immigration coefficient $m_{k}$ (Hubbell 2001) can

239 then be computed as

$$
m_{k}=\frac{b_{l}}{b_{l}+b_{k}-1}
$$

Now we can compute the recruitment probability of a species $i$ in the assemblage $k$ given the neutral parameters $R_{\text {Dispersal }_{i M}}, N_{i k}, N_{i M}$, and $m_{k}$ (Sokol et al.

243 2015), as follows:

$$
R_{\text {Neutral }_{i k}}=m_{k} \cdot R_{\text {Dispersal }_{i M}}+\left(1-m_{k}\right) \cdot N_{i k}
$$

Finally, we compute the recruitment probability of any species $i \in q$ in any assemblage $k \in M$ that considers both niche and neutral processes, as

$$
R_{i k}=R_{\text {Niche }_{i k}} \cdot R_{\text {Neutral }_{i k}} \cdot
$$

248 The definition of $R_{i k}$ provides an integrative solution to model species assembly patterns in local assemblages that allow estimating the strength of niche and neutral process determining the dynamics of biological diversity across space. Yet, the evaluation of the imprint left by niche evolution on species assembly, and ultimately on biological diversity, is still lacking. Over the next section we present a solution to fill this gap.

\section{Niche evolution and species assembly across environmental gradients}

254 The Ornstein-Uhlenbeck (OU) model of trait evolution (Hansen 1997) allows

255 parameterizing the strength of the adaptation rate $\alpha$ of species niches evolving towards an optimum niche condition $\theta$, as follows:

$$
d y_{i}(t)=\alpha\left[\theta-y_{i}(t)\right] d t+\sigma d B(t)
$$


258 For any $\theta$ and given the niche position $y_{i}(t)$ of species $i$, adaptation rate $\alpha$ is a force that 259 deterministically decreases the fitness of $y_{i}(t)$ as $\theta-y_{i}(t) \neq 0$. As $\alpha \rightarrow 0$, the strength of

260 adaptation rate decreases, and trait divergence among species is increasingly modeled

261 by the stochastic component of the OU model $(d B[t])$, which is a stochastic white-noise

262 process showing Gaussian distribution $(N[0, d t)]$, Brownian motion), and standard

263 deviation $\sigma$, which indicates the amplitude of niche position variation among species

264 (Butler and King 2004; Hansen et al. 2008). Therefore, under low $\alpha$ values, species

265 niche position evolves under strong phylogenetic inertia, generating high phylogenetic

266 signal in species niche positions (Blomberg and Garland 2002). On the other hand, as $\alpha$

$267 \rightarrow \infty$, species niches tend to evolve faster from the ancestral state $\left(y_{i \text { anc. }}\right)$ towards $\theta$, and

268 the imprint of phylogenetic inertia on species niches tends to be erased. The rate of

269 niche evolution towards $\theta$ can be estimated as a phylogenetic half-life (Hansen 1997;

270 Hansen et al. 2008), which indicates the amount of time units needed to move half the

271 distance between the ancestral state and the optimum condition, and is calculated as

$$
\mathrm{t}_{1 / 2}=\frac{\ln 2}{\alpha}
$$

Note that $t_{1 / 2}$ is expressed in time units, which means that the effect $\alpha$ exerts on niche evolution depends on the timespan between $y_{i}$ anc. and $\theta$. If the timespan is long enough (say, 200 million years), even a small $\alpha$ value (say, $\alpha=0.01$ ) may generate a moderate movement towards $\theta\left(t_{1 / 2}=69.3\right)$. Nonetheless, if the evolutionary timespan is shorter than $\cong 70$ million years, the phylogenetic half-life corresponding to $\alpha=0.01$ will be too slow to produce significant niche evolution towards the niche optimum, and therefore niche evolution may show phylogenetic inertia not quite distinguishable from Brownian motion $\left(\alpha=0, t_{1 / 2}=+\infty\right)$. Thus, the expected value of a niche $y_{i}$, evolving under OU model during a time $t$, results from a deterministic process modulated by the adaptation rate $\alpha$ that forces $y_{i}(t)$ to converge towards the optimum niche condition $\theta$, and a stochastic, Brownian process, as follows:

$$
\mathrm{E}\left[y_{i}(t)\right]=\theta\left(1-e^{-\alpha t}\right)+y_{i \text { anc. }} e^{-\alpha t} .
$$

The critical step is to translate the OU parameters $\alpha$ and $\theta$ into quantities that can be estimated from assemblages described by species abundances or occurrences, an environmental variable associated with species assembly, and a phylogenetic tree. In a metacommunity $M$ composed by $k$ local assemblages defined by $q$ species belonging to 
a monophyletic lineage, those local assemblages with the highest levels of biological diversity can be considered as offering the best environmental condition $x$ to most species in $q$ (Enquist et al. 2015; Violle et al. 2007), and therefore it is likely to be an appropriate estimator of $\theta$. We might then take $x$ as an empirical estimate of $\theta$, or $x_{\theta}$.

Niche filtering is expected to be stronger in assemblages with environmental condition deviating from $x_{\theta}$, leading to lower species diversity. Differently from $x_{\theta}$, which can be estimated from data, the parameter $\alpha$ cannot be directly assessed, but its posterior distribution can be estimated by means of rejection-based Approximate Bayesian Computation (Beaumont 2010; Csilléry et al. 2010). We explain this in detail in the next section.

For now, it is important to understand what the parameter $\alpha$ can inform about the role of niche evolution on species assembly patterns. If $\alpha \rightarrow 0$, we may conclude that niche evolution was mainly a stochastic Brownian process, which means that the adaptation rate towards $x_{\theta}$ was very weak along niche evolution, insufficient to break the phylogenetic signal of the ancestral niche of species. Under such evolutionary scenario, niche divergence between species will be a function of the time since their evolutionary divergence, leading to strong phylogenetic covariation in species niches. Furthermore, the total variance in the niche position will be high, which means that niche positions of species will be more loosely distributed across the upper and lower limits of the environmental condition $x$. Niche-based assembly processes in local communities will tend to be phylogenetically structured, while differences in diversity values among assemblages will be not remarkable. As $\alpha \rightarrow \infty$, adaptation rate of species

311 niches towards $x_{\theta}$ becomes faster along niche evolution, so that any track of

312 phylogenetic signal in the niche vanishes along evolution (Cooper et al. 2016). Niche

313 divergence between species will be constrained by the optimum niche condition, with

314 values approaching $x_{\theta}$ as $\alpha$ increases, decreasing the total variance of the niche position

315 among species. In local assemblages showing environmental conditions close $x_{\theta}$, steep

316 diversity peaks are expected to occur.

\section{Estimating niche and neutral parameters underlying species diversity gradients}

\section{using Approximate Bayesian Computation}

319 Consider that assemblages in $M$ are distributed across an environmental gradient

320 defined by a single environmental condition, or multiple conditions summarized in a

321 single factor or principal component $x$. We may estimate $x_{\theta}$ as the environmental 
322 condition interval that maximizes species diversity in $M$. In turn, whenever the niche

323 position $y_{i}$ of a given species in $M$ approaches $x_{\theta}$, the probability of occurrence of that

324 species in the assemblage increases, conditioned to the niche positions of all other

325 species in the assemblage. The adaptation rate of $y$ along the evolutionary history (i.e.

326 OU's $\alpha$ ) is a niche-assembly parameter that can be estimated given the species diversity

327 variation in $M, x$, the spatial distribution of local assemblages in $M$, and the

328 phylogenetic relatedness among species distributed across $M$. By doing so, we can

329 reveal how strong is phylogenetic signal in niche position of species. On the other hand,

330 species diversity in a local assemblage may be limited by the dispersal capacity of

331 species. As we have seen before, this depends on the spatial distance between sites $(r)$

332 and the slope $w$ of the dispersal kernel, in complement to niche characteristics of species

333 (Gravel et al. 2006). Therefore, estimating $w$ allows quantifying the influence of

334 dispersal limitation on the distribution of species diversity across $M$.

335 To estimate niche (OU's $\alpha$ ) and neutral (slope $w$ ) parameters associated with the

336 distribution of species diversity, we developed an Approximate Bayesian Computation

337 (ABC) algorithm (Beaumont et al. 2002; Csilléry et al. 2010) that works as

338 schematically represented in figure 1. 


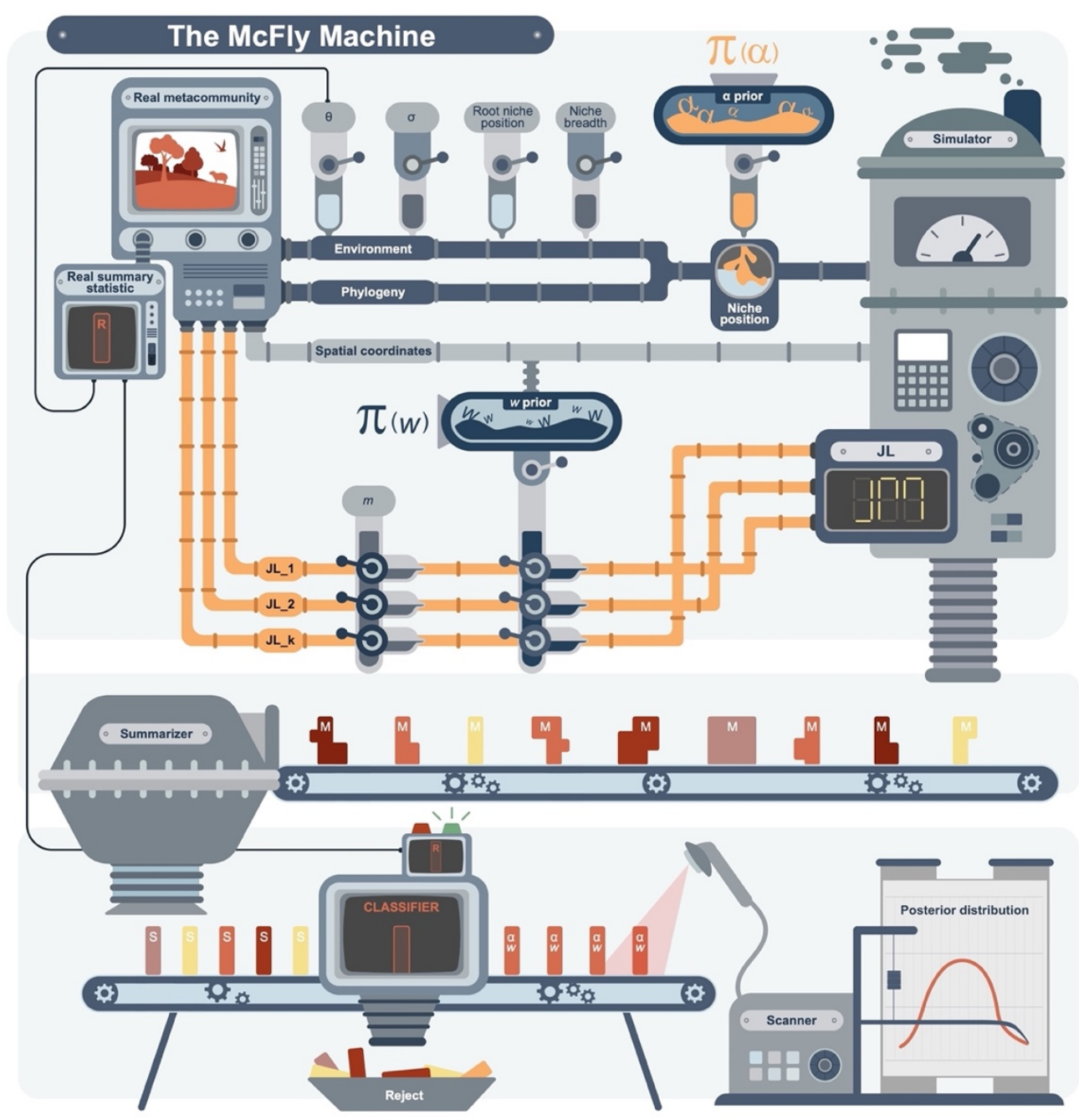

341 Figure 1: Schematic representation of $m c f l y$, an Approximate Bayesian Computation framework

342 developed to estimate the posterior distribution of adaptation rate (OU's $\alpha)$ and dispersal kernel slope $(w)$ parameters driving species diversity gradients along an environmental gradient. 1. The real metacommunity provides the empirical measures of species diversity, the real summary statistic $(R) .2$. Environment is a dimension of species niches that provides the optimum niche $(\theta)$, variance $(\sigma)$ and root niche position parameters necessary to simulate species niche based on the phylogeny, according to Ornstein-Uhlenbeck evolutionary model. We also define niche breadth in the simulation based on environment. 3. A prior distribution of OU's $\alpha$ is used to simulate species niche positions based on environment parameters and phylogeny. 4. Spatial coordinates of $k$ sites are used to define the prior distribution of slope $w$; together with immigration rate $(m)$, number of individuals at $k$ sites $\left(J_{L_{-}} k\right)$ and the total number of individuals in $M\left(J_{M}\right)$ provide the neutral parameters of simulations. 5. Metacommunities are simulated based on niche and neutral parameters (Simulator). 6. Each metacommunity simulation provides species diversity measures (Summarizer) taken as the simulated summary statistics (S). 7. Simulated diversities $(S)$ are classified based on a pre-defined minimum correlation threshold (tolerance) to the observed diversity $(R)$. Every $S$ object not discarded (Reject) is then scanned to record its OU's $\alpha$ and slope $w$ to provide a posterior distribution of adaptation rate $(\alpha)$ and dispersal limitation $(w)$, respectively. 
360 An additional function that helps users to define the appropriate tolerance threshold

361 ('define_tolerance') is also available in the package, as well as a working example to

362 illustrate how the user can set all the parameters needed to run 'Mcfly' function. The

363 example can be easily accessed in R by typing vignette("mcfly_vignette”).

\section{The 'simulator engine'}

365 The simulation framework implemented in mcfly package derives from the individual-

366 based simulation protocol developed by Sokol et al. $(2015,2017)$ and available in the R

367 package MCSim (available at https://github.com/sokole/MCSim). Accordingly, sets of

368 assemblages are simulated based on lottery colonization and recruitment dynamics,

369 following niche and neutral mechanisms (Gravel et al. 2006), as explained in the section

$370 \quad$ Parameterizing niche and neutral processes determining species assembly.

371

372

373

374

375

376

377

378

379

380

381

382

383

384

385

386

387

388

389

390

391

\section{Metacommunity dimensions and species pools}

Simulated metacommunities $M_{\text {Sim }}$ show the same number of sites of the observed metacommunity $M$. If $M$ contains information on species abundances, the number of individuals in each simulated assemblage equals the number shown in the respective observed local assemblage. Because the original simulation protocol of MCSim is individual-based, when only species incidence (presence/absence) data is available the simulation performed in mcfly addresses 100 individuals to each species present in each local assemblage prior to the simulation of $M_{\text {Sim }}$. In the end, $M_{\text {Sim }}$ is transformed to an incidence matrix.

Noteworthy, the phylogenetic tree used to build $M_{\text {Sim }}$ should be as complete as possible to increase the quality of niche simulations, which means it should not be pruned to keep only the species present in $M$. In all simulations, the species pool of $M_{\text {Sim }}$ will be the same defining $M$, independently of the size of the species pool included in the phylogeny. According to Sokol et al. (2015), recruitment patterns in $M_{\text {Sim }}$ stabilize after 30 simulation time steps. By default, we set simulations to stop after 50 timesteps.

\section{Niche prior and other parameters}

At each simulation run, the niche position of each species is simulated for all species included in the phylogenetic tree using the function 'rTraitCont' of the package ape (Paradis and Schliep 2019) as a continuous trait $y$ following OU model. The adaptation rate parameter $\alpha$ of niche simulation is randomly sampled from a prior distribution, with predefined size and distribution mode. The minimum value of the $\alpha$ parameter is set to 
392 correspond to the half-life $\left(t_{1 / 2}\right.$, see eq. 9) equal to the total timespan of the phylogeny.

393 For a phylogeny with timespan $=30 \mathrm{Myr}$, the minimum $\alpha$ value will be 0.023 , since

$$
\mathrm{t}_{1 / 2}=\ln (2) / 0.023=30 \text {. }
$$

395 The maximum $\alpha$ parameter value corresponds to $t_{1 / 2}=1 / 30 \cdot$ timespan. Thus, for that same timespan $=30 \mathrm{Myr}$, the maximum $\alpha$ value $=0.693$, since

$$
\mathrm{t}_{1 / 2}=\ln (2) / 0.693=(1 / 30) \cdot 30=1 \text {. }
$$

In the example above, the range of $\alpha$ parameters between the minimum and maximum limits expresses a broad variation in phylogenetic signal. Indeed, we observe

400 an asymptotic decay in phylogenetic signal, estimated by Blomberg's K (Blomberg and

401 Garland 2002), with increasing OU's $\alpha$ values (fig. 2). Considering the $\alpha$ parameter

402 interval ranging between 0.023 and $0.693, \mathrm{~K}$ statistics is shown to vary between 0.983

403 and 0.137 , respectively. Further, the lower $95 \%$ credibility limit for OU's $\alpha$ values

404 estimated from vectors randomly drawn from Gaussian distribution (white noise)

405 reveals that niche positions showing $\alpha$ values higher 0.628 cannot be distinguished from

406 white noise variables and show very low phylogenetic signal $\leq 0.165$. Therefore, we

407 conclude that the range of prior $\alpha$ values defined in the simulation algorithm is

408 appropriate. Note that the simulation does not rescale the branch lengths of phylogenetic

409 tree. Therefore, the prior distribution of OU's $\alpha$ values will differ from the above

410 whenever the timespan differs from $30 \mathrm{Myr}$, and therefore the interpretation of results

411 must consider the timespan of the phylogenetic tree under analysis. By default, $m c f l y$

412 generates a uniform distribution of the $\alpha$ prior, but the user may opt for generating a

413 prior $\alpha$ with half-life values uniformly distributed, instead. In the next sections we

414 provide a sensitivity analysis to evaluate the implications of the $\alpha$ prior distribution for

415 the posterior distribution of OU's $\alpha$ parameter. 
417

418 Figure 2: Relationship between Blomberg's K statistic and the adaptation rate parameter of Ornstein-

Uhlenbeck model (OU's $\alpha$ ) for simulated niche positions computed for 1,000 sets of 50 species each (black circles). Gray areas indicate lower and upper credibility intervals (95\%) for Blomberg's K and OU's $\alpha$ values estimated from white noise vectors $(N[0,1])$.

Another critical niche parameter to be defined is the optimum niche position $\left(x_{\theta}\right)$. In our framework, $x_{\theta}$ is empirically defined as the values of the environmental condition $e$ showing the highest species diversities in $M$. In $m c f l y$, all $e_{k}$ values corresponding to $\geq$ the $5 \%$ highest species diversity values will constitute a vector of $x_{\theta}$ values. At each metacommunity simulation process, a single $x_{\theta}$ value is randomly drawn from that vector and taken as the optimum niche. Thus, it is important to note that the more unimodal is diversity distribution across $e$, the more homogeneous will $x_{\theta}$ among different $M_{\text {Sim }}$, which is likely to decrease the credibility intervals of the posterior distribution of OU's $\alpha$ and slope $w$ parameters. Moreover, the variance of the niche position $(\sigma)$ was empirically defined as $\sigma=\sqrt{\operatorname{standard~deviation~}(x)}$, and root node value $=\bar{x}$.

\section{Neutral prior and other parameters}

The spatial coordinates of each assemblage in $M$ are used to compute pairwise distances $r$ between local assemblages in $M$, which are rescaled to vary between 0 and 1 . As we have seen in equation $3, r$ is a component defining the probability of a species to disperse from a site to another, whose effect on dispersal limitation is modulated by the slope $w$, the neutral assembly parameter of interest in our framework. It is important to note that the range of variation of $w$, which expresses the strength of dispersal limitation, depends on the shape of the spatial distribution of sites. When sites are

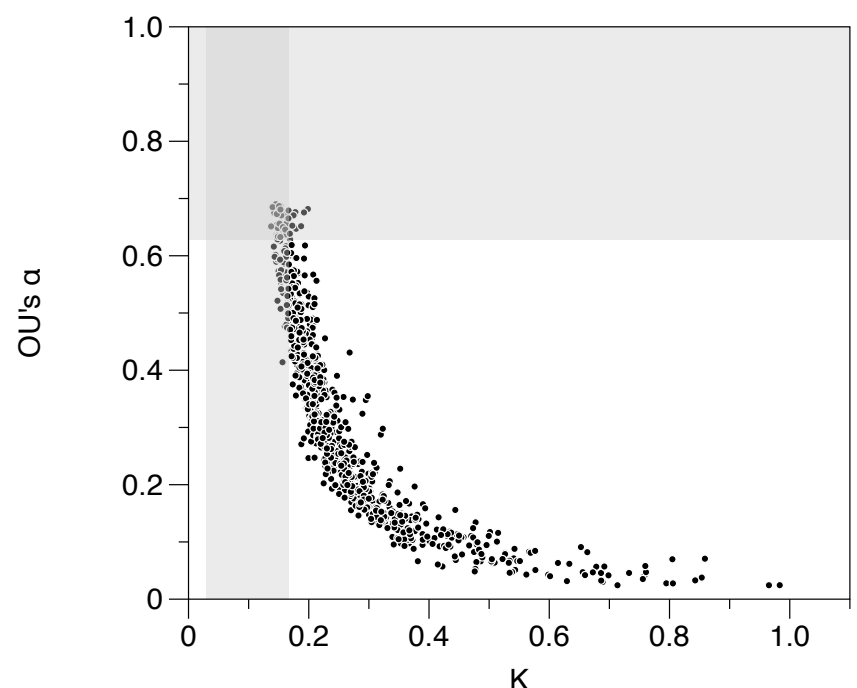


evenly distributed across the space, as in a cell grid, $w$ slopes must vary over broader scales to capture dispersal limitation over shorter distances, and vice-versa. Thus, to

443 build a prior distribution of slope $w$, we first defined the maximum distance $r$ between

444 sites enough to connect all sites in $M$ by means of a minimum spanning tree (Legendre and Legendre 2012). This threshold distance provides information on how far two sites should be to each other to dispersal limitation to become relevant and is widely used in spatial analysis (Borcard and Legendre 2002). Then, we simulated a uniform distribution of dispersal probabilities between sites ( $\delta_{\mathrm{lk}}$, see eq. 3 ), varying between 0 and 1, and with a predefined size. Based on equation 3, we then computed the slope $w$ corresponding to every simulated $\delta_{\mathrm{lk}}$ for a hypothetical pair of sites located at distance $r$ to each other. By doing so, we build the prior distribution of slopes $w$, which is not uniform itself, but decays logarithmically as $w$ increases.

As we have seen in eq. 5 , the probability of immigration in each assemblage $(m)$ has also to be defined prior to the simulation, which is not trivial in most cases. The user may opt for indicating an arbitrary value; the default value in mcfly is 0.5 , which means that the simulation allows a half of the individuals to be replaced at each timestep of the simulation process. Alternatively, the user may inform empirically estimated $m$ for each site, whenever these data are available.

460 Our simulation framework summarizes species diversity in the local assemblages of $M$ and $M_{\text {Sim }}$ using Rényi entropy (Rényi 1961), which is a flexible measure that generalizes widely used diversity measures, such as Shannon and Simpson diversity indices (Anand and Orlóci 1996). Rényi entropy $\left(\mathrm{H}_{\text {order }}\right)$ is computed as follows:

$$
\mathrm{H}_{\text {order }}=\frac{\log _{2} \sum_{i=1}^{q} \mathrm{p}_{i}^{\text {order }}}{1-\text { order }} .
$$

Accordingly, $q$ is the number of species present in the community, $p_{\mathrm{i}}$ is the proportional abundance of each species $i$ in the community, and order is a scalar that modifies the sensitivity of entropy to variation in species abundances (evenness). In this study, we analyzed three entropy orders: 1, 2, and 12. For entropy order $=1$, Rényi entropy is equivalent to Shannon diversity index, while entropy order $=2$ assumes values

470 equivalent to Simpson index (Anand and Orlóci 1996). Higher entropy orders increase 471 the importance of evenness in the diversity index, which tends to stabilize for entropy 
order $>12$ (Anand and Orlóci 1996). For datasets characterizing sites by species incidences (presence/absence data), Rényi entropy is equivalent to species richness (or entropy order $=0$ ), independently of the entropy order used.

\section{The posterior distribution of adaptive rate $(\alpha)$ and dispersal limitation ( $w$ )}

476 Since species diversity is computed for $M$ and all $M_{\text {Sim }}$ datasets, the correlation between observed and simulated diversities is a way to evaluate how similar are observed and simulated metacommunities. This step involves defining a threshold correlation value below which metacommunities are rejected. In $\mathrm{ABC}$ analysis, a tolerance value is usually defined, and expresses the maximum distance tolerated between observed and simulated summary statistics (Beaumont 2010). In $m c f l y$, the tolerance expresses the complement of the correlation $(1$ - correlation) and must be informed prior to the simulations. The remaining $M_{\text {Sim }}$ datasets surpassing the tolerance threshold provide their corresponding OU's $\alpha$ and slope $w$ parameters to a posterior distribution of niche and neutral parameters underlying diversity patterns in $M$, respectively. A highest posterior distribution (HPD) provides the credibility intervals of those parameters (Turkkan and Pham-Gia 1993).

\section{Evaluating $m c f l y$ performance using simulated metacommunities}

To evaluate the performance of $m c f l y$ we simulated 2,000 metacommunities $M$ using the function 'metasim' of the package MCSim (Sokol et al. 2017; Sokol et al. 2015), each of them showing 50 local assemblages, with known niche and neutral parameters, which allowed to evaluate the efficiency of the proposed simulation approach to estimate them.

Prior to metacommunity simulation, phylogenetic trees containing 50 species were simulated using the function 'sim.bdtree' of package geiger (Harmon et al. 2008), assuming homogeneous birth-death processes across all lineages. Speciation followed purely stochastic birth (speciation rate $=0.1$, extinction rate $=0$ ). The number of lineages increased exponentially with time. Evolutionary timespan was fixed as $30 \mathrm{Myr}$. These phylogenies defined the species pools used to perform all further simulations. All 50 species in each phylogeny were allowed to occur in at least one out of 50 communities representing a metacommunity $M$. 
environmental variable $x$ was simulated from a uniform distribution $(\mathrm{U}[0,100])$, and its mean value $(\bar{x})$ was defined as the optimum niche position $(\theta)$. OU's $\alpha, \bar{x}$ and other niche parameters described in the section Niche prior and other parameters were then used to simulate the niche positions $y_{i}$ of species ranging between 0 and 100 based on OU method of the 'rTraitCont' function of package ape (Paradis and Schliep 2019). For metacommunity simulation, species niche breadth ( $s_{i}$, eq. 1$)$ was fixed as 10 , which confers reasonable niche breadth to species (Duarte et al. 2018; Peres-Neto et al. 2012). distribution $(\mathrm{U}[0,1])$. The number of individuals per local assemblage and the total number of individuals in $M$ were defined as, respectively, $J_{k}=1000$ individuals, $J_{M}=$ $1000 \times 50$ sites $=50,000$ individuals. The probability of immigration ( $m$, see eq. 5 ) was defined as 0.5 . The frequencies of species across $M$ were extracted from a multinormal distribution ranging between 0.1 and 0.4 that was further normalized based on $J_{M}$. Species colonization or recruitment across $M$ stopped after 50 simulation time steps. Noteworthy, in some simulations, a low number of species was capable of colonizing at least one local assemblage in $M$, and therefore gamma diversity in $M$ varied among the simulations. We analyzed only $M$ datasets containing, at least, 20 species distributed across local assemblages. We divided the 2,000 $M$ datasets into five subgroups of 400 metacommunities, each of them simulated under different dispersal limitation strengths $(w=0,1,5,15$ or 100 , see Neutral prior and other parameters). Thus, the real $w$ slope of each simulated $M$ was known. After metacommunity simulation, each $M$ dataset was converted into an incidence matrix describing species occurrences in each local assemblage.

After defining $M$ datasets, we submitted each metacommunity to ABC analysis using $m c f l y$ to evaluate the suitability of the framework to estimate the posterior distribution of the adaptive rate of niche positions (OU's $\alpha$ ) and dispersal limitation strength (slope $w$ ). Prior to ABC analysis, the 2,000 metacommunities $M$ were split into two groups containing 1,000 metacommunities each. In the first group, the prior distribution of OU's $\alpha$ used in the ABC algorithm was defined as a uniform distribution of $\alpha$ values ('uniform' $\alpha$ prior). In the second group, $\alpha$ prior distribution was defined based on a uniform distribution of $\alpha$ half-lives ('half-life' $\alpha$ prior), and the corresponding $\alpha$ values were then taken as OU's $\alpha$ prior (see Niche prior and other parameters). The maximum sample size of $\alpha$ and $w$ priors used to analyze each metacommunity $M$ was defined as 14,400 simulations of $M_{\text {Sim }}$ datasets, while the 
posterior sample size was defined as 240 . Since we had no previous information about the appropriate tolerance value (see previous section), prior to $\mathrm{ABC}$ we computed correlations between the summary statistics computed for each $M$ dataset and for 480 $M_{\text {Sim }}$ datasets to obtain a correlation distribution. The complement of the $95^{\text {th }}$ percentile of the respective correlation distribution was then taken as the tolerance value in $\mathrm{ABC}$ performed for each metacommunity $M$.

At each simulation step, $m c f l y$ simulated $M_{\text {Sim }}$ under predefined adaptation rate (OU's $\alpha$ ) and dispersal limitation (slope $w$ ), computed the summary statistics (Rényi entropy of order 1), and correlated it to the real summary statistics computed from $M$. Whenever the complement of correlation was $\leq$ the tolerance limit, $\alpha$ and $w$ parameters were recorded in the posterior distribution of $\alpha$ and $w$ parameters. After the algorithm reached either the posterior sample size or the maximum prior size (in cases where the posterior sample size was not reached), the median values of each parameter in the posterior distribution were recorded.

\section{Statistical performance of $\mathrm{ABC}$ based on simulated datasets}

We evaluated the statistical performance of the ABC approach using structural equation modelling based on $d$-separation tests (Shipley 2000), which estimates the validity of causal models using Fisher's $\mathrm{C}$ as test statistic that follows a chi-square distribution with $2 k$ degrees of freedom, where $k$ is number of independence relationships necessary to evaluate the model given the number of variables and the number of causal relationships proposed in the model (Shipley 2000). Accordingly, the probability of the null hypothesis of the fitted model to correspond to expected causal relationships indicates the overall validity of the proposed model. Path coefficients $(p)$ linking variables in the causal model were estimated using ordinary least squares (OLS). The analysis was performed using the 'psem' function of the package piecewiseSEM (Lefcheck 2016).

We built the causal models considering first the effects of both real OU's $\alpha$ and $w$ on the number of species in $M$ (which varied across simulations, as some species were lost during the assembly), and on the median of the posterior distribution of both

568 OU's $\alpha$ and $w$. Furthermore, we evaluated the influence of the prior OU's $\alpha$ mode

569 ('uniform' or 'half-life') on the median of the posterior distribution of OU's $\alpha$. Finally, 570 the influence of the number of species in $M$ on both was the median of the posterior 
572 and posterior OU's $\alpha$ by the respective half-life and tested similar causality

573 relationships. Prior to causal testing, half-life and $w$ slopes were log transformed.

574 We also evaluated causal relationships within each prior OU's $\alpha$ mode. These

575 models were tested because we found that the effect of prior OU's $\alpha$ mode on the

576 median of the posterior distribution of OU's $\alpha$ was stronger than the effect of the real

577 OU's $\alpha$ (the same occurred in the half-life model). Evaluating causal relationships

578 within each prior OU's $\alpha$ mode enabled us to perform a sensitivity analysis of the ABC

579 approach to the prior OU's $\alpha$.

580 In all causal models, we considered that the residuals of the median of the

581 posterior distribution of OU's $\alpha$ and $w$ slope were correlated to each other.

A study case: phyllostomid bats

583 We analyzed the influence of niche evolution and dispersal limitation on the distribution

584 of species diversity of the Phyllostomidae, a species-rich and ecological diverse family

585 of bats. Phyllostomid bats are predominantly distributed in the Neotropics, from

586 California to northern Argentina and Chile (Fleming et al. 2020). The family has 222

587 known species (Simmons and Cirranello 2020), displaying a large array of dietary and

588 morphological specializations to feed on fruits, nectar, and other parts of plants, as well

589 as on insects, vertebrates, and blood (Baker et al. 2012; Fleming et al. 2020; Monteiro

590 and Nogueira 2011; Rossoni et al. 2019). The remarkable diversity of their ecological

591 features mirrors a strong latitudinal and environmental gradient of species richness

592 (Pereira and Palmeirim 2013; Villalobos and Arita 2010), which makes this family

593 particularly appropriate for our purpose. We gathered geographic range maps for

594 phyllostomid species from IUCN database (IUCN 2020), and defined species

595 assemblages based on species incidences in a grid composed by $110 \times 110 \mathrm{~km}$ cells

596 distributed across the Neotropical region strictu sensu (see Maestri and Duarte 2020;

597 Morrone 2014). Phylogenetic relationships among species were taken from the

598 consensus phylogenetic hypothesis proposed by Shi and Rabosky (2015), the most

599 comprehensive bat phylogeny available to date. The complete Chiroptera phylogeny

600 was dated using 24 fossil calibration points distributed along the whole timespan of the

601 tree. Pruning geographic data given available genetic information resulted in a dataset

602 of 136 species ( $c$ a. $62 \%$ of total phyllostomid richness) distributed across 1,338

603 assemblages in the Neotropical region. The total timespan of the pruned phylogeny was

$604=34$ Myr. 
We computed entropy of order 1 for sites described by phyllostomid species and analyzed its association with each of four climatic variables (mean annual temperature, temperature seasonality, mean annual precipitation and precipitation seasonality) and two topographical variables (mean altitude and altitudinal range variation). Climatic variables were taken from the WorldClim2 database (Fick and Hijmans 2017), and topographic variables were taken from NASA (http://neo.sci.gsfc.nasa.gov/).

611 Phyllostomid entropy was strongly negatively related to temperature seasonality $(r=$

612 0.91), corroborating results obtained elsewhere (Stevens 2011), and was taken as the 613 niche condition $(x)$ in further analysis.

614 Then, based on the species by sites matrix (assemblages), the spatial coordinates

615 of the sites, the niche condition (precipitation seasonality), and phylogenetic

616 relationships among species, we were able to estimate the posterior distribution of the

617 adaptive rate of species niche positions (OU's $\alpha$ ) and dispersal limitation strength (slope

$618 w$ ) underlying the distribution of species diversity across phyllostomid assemblages

619 using $\mathrm{ABC}$ analysis implemented in mcfly. To simulate niche positions, we defined

$620 \sigma=\sqrt{\text { standard deviation }(y)}$, root node value $=\bar{y}$. The probability $m$ of new colonizers to

621 arrive at simulated assemblages $=0.5$. Niche breadth $\left(s_{i}\right)$ was set to 10 . Species

622 colonization or recruitment in each set of simulated communities stopped after 50

623 simulation time steps. We set the maximum sample size of OU's $\alpha$ (uniform mode) and

$624 w$ priors to 36,000 , and the size of the posterior sample to 480 . The tolerance in $\mathrm{ABC}$ 625 analysis $(1-r)$ was set to 0.1 .

\section{Results}

\section{Simulated datasets}

628 The results of global causal model analyzing factors influencing the posterior

629 distribution of OU's $\alpha$ and $w$ slope are shown in figure $3 A$. The overall model fit

630 (Fisher's $\mathrm{C}=2.67, P_{\mathrm{df}=4}=0.62$ ) indicated that the model was causally valid. The

631 median of the posterior distribution of OU's $\alpha$ was mostly explained by the OU's $\alpha$

632 prior mode $(p=0.69)$, and to a lesser extent by the real OU's $\alpha(p=36)$ and the real $\mathrm{w}$

633 slope $(p=0.2)$. The number of species in the metacommunity did not affect the median

634 of the OU's $\alpha$ posterior distribution $(p<0.01)$. On the other hand, the median of the

635 posterior distribution of $w$ was mostly explained by the real $w$ slope $(p=0.85)$.

636 Replacing the median of the posterior distribution of OU's $\alpha$ by half-life (fig. $3 B$ ) did

637 not change the overall architecture of causal relationships. The overall model remained 
638 valid (Fisher's $\mathrm{C}=1.18, P_{\mathrm{df}=4}=0.88$ ); noteworthy, the strength of the path coefficient

639 linking the real half-life to the median of the posterior distribution of OU's $\alpha$ increased

$640(p=0.42)$, while the latter was still mostly explained by the OU's $\alpha$ prior mode $(p=-$

641 0.74). All other causal relationships behaved similarly to the observed in the previous

$642 \operatorname{model}($ fig. $3 A$ ).

643 Bivariate relationships among real OU's $\alpha$, half-life and $w$ slope and the

644 corresponding median values of the posterior distribution of OU's $\alpha$, half-life and $w$

645 slope are shown in figure 4. In figures $4 A, C$ and $E$, we observe relationships obtained

646 when the prior distribution of OU's $\alpha$ was uniform, whereas figures $4 B, D$ and $F$ show

647 the relationships obtained using the half-life derived prior distribution of OU's $\alpha$.

648 Exploring these bivariate relationships suggests that using the uniform prior distribution

649 of OU's $\alpha$ produce stronger associations between real OU's $\alpha$ or half-life and the

650 median posterior distribution of OU's $\alpha$ (fig. $4 A$ ) or half-life (fig. $4 C$ ), respectively, than

651 the corresponding relationships based on half-life derived OU's $\alpha$ prior (figs. $4 B, D$ ).

652 But even using the uniform OU's $\alpha$ prior, the dispersion of the median posterior

653 distribution of both OU's $\alpha$ and half-life tended to increase towards higher real OU's $\alpha$,

654 which should possibly lead to lower reliability of the posterior distribution of the $\alpha$

655 parameter under higher real OU's $\alpha$. Such tendency was minimized when the

656 association between log-transformed real half-life and median of the posterior

657 distribution of half-life was considered, as we also found a linear relationship between

658 those variables when the uniform prior distribution of OU's $\alpha$ was used (fig. 4C).

659 Further, the association between real $w$ and the median posterior distribution of $w$ slope

660 was not affected by OU's $\alpha$ prior mode, as expected (figs. $4 E, F$ ).

661

As the OU's $\alpha$ prior mode was found to be the best predictor of the median

662 posterior distribution of OU's $\alpha$, we built the causal models considering only the tests

663 performed using each OU's $\alpha$ prior mode ('uniform' or 'half-life'). Figures $5 A, B$ show

664 models built considering the uniform OU's $\alpha$ prior, while figures $5 C, D$ show models

665 built for the half-life OU's $\alpha$ prior. In general, models using the half-life derived OU's $\alpha$

666 had worse overall fit than models using the uniform OU's $\alpha$ prior, which had lower

667 Fisher's $\mathrm{C}$ statistics than the former. Within the models using the uniform OU's $\alpha$ prior,

668 the most adequate scenario was obtained for the scenario considering log-transformed

669 real half-life explaining the median posterior distribution of half-life, considering the

670 uniform OU's $\alpha$ prior ( $p=0.67$; fig. $5 B$ ). Furthermore, that model showed the lowest

671 correlation between the median of the posterior distribution of OU's $\alpha$ and $w$ slope. 
A)

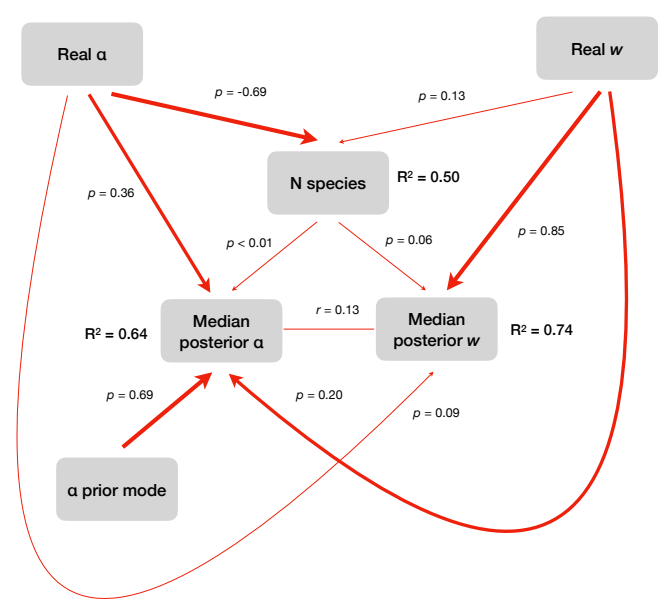

B)

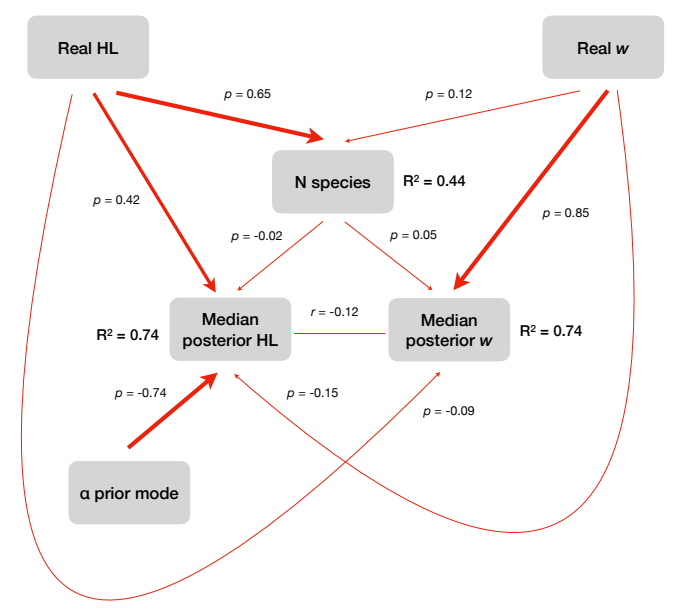

673 Figure 3: Causal models relating real OU's $\alpha$ or half-life, real $w$ slope, OU's $\alpha$ prior mode and number of species in 2,000 simulated metacommunities on the median of the posterior distribution of OU's $\alpha$ of half-life and $w$ slope. Causal relationships were estimated using ordinary least squares. Half-life and $w$ slopes were log-transformed prior to analysis. Validity of overall models was tested using d-separation tests (Shipley 2000). $A$ ) OU's $\alpha$ model (Fisher's C=2.67, $P_{\mathrm{df}=4}=0.62$ ). $B$ ) Half-life model (Fisher's $\mathrm{C}=$

678 $\left.1.18, P_{\mathrm{df}=4}=0.88\right)$. Arrow width indicates the strength of the respective causal relationship.

679 

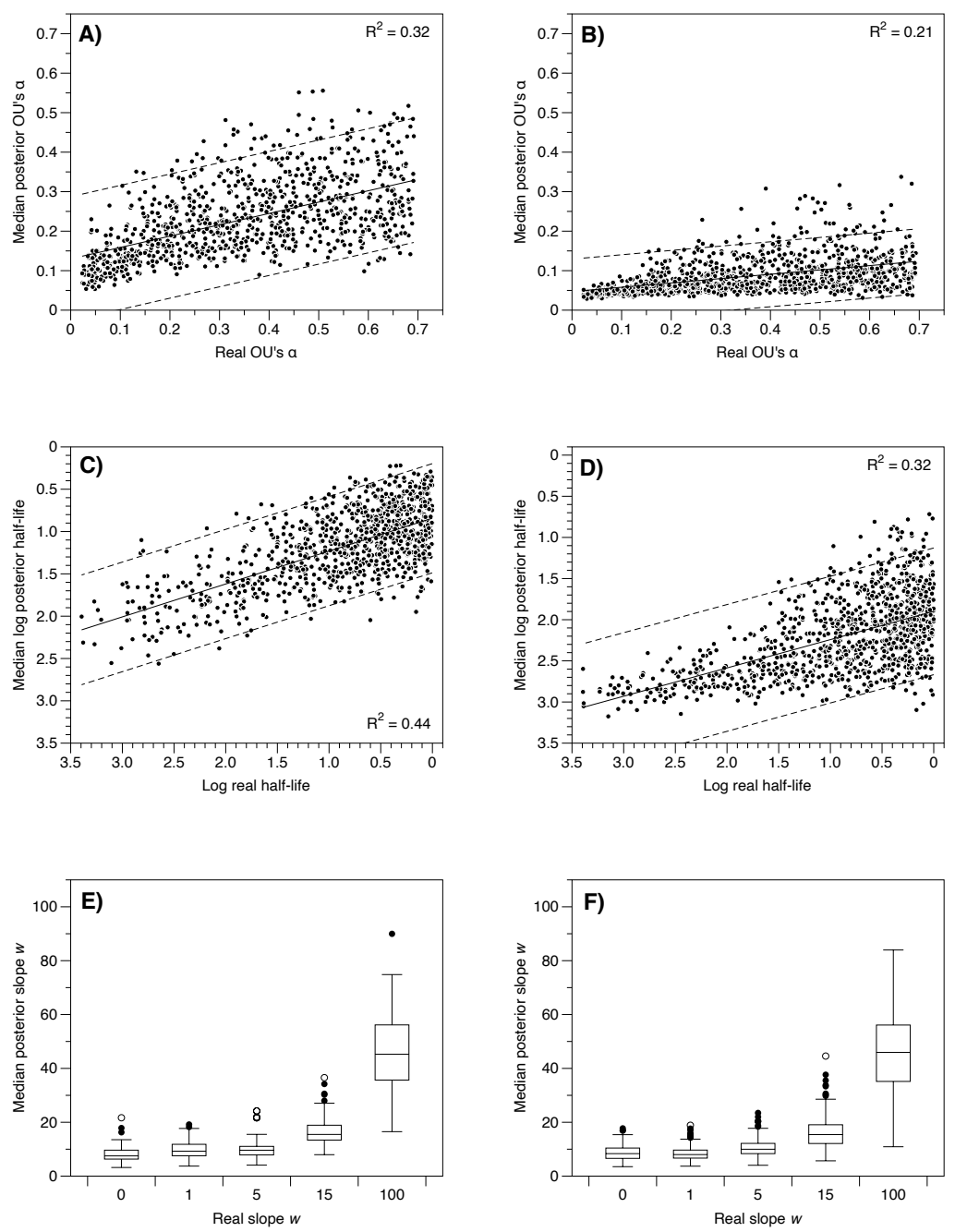

$681 \quad$ Figure 4: Bivariate relationships between real niche (OU's $\alpha$ or half-life) or dispersal ( $w$ slope) parameters of simulated metacommunities and the corresponding median of the posterior distribution of

683 OU's $\alpha$, half-life and $w$ slope. $A, C, E$ ) Uniform OU's $\alpha$ prior. $B, D, F)$ Half-life derived OU's $\alpha$ prior.

684 Dashed lines delimit confidence intervals (95\%) of predictions.

685 
A)

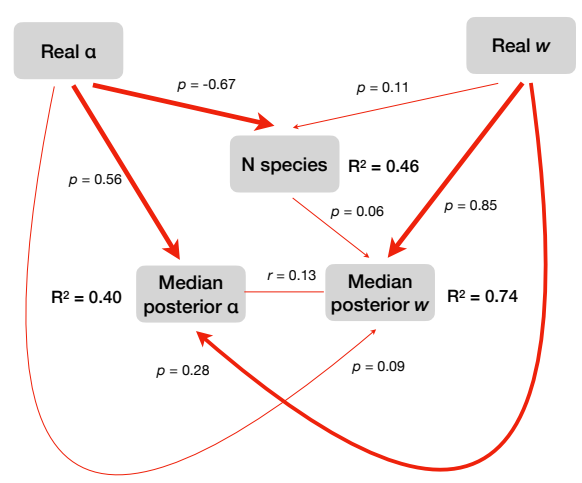

C)

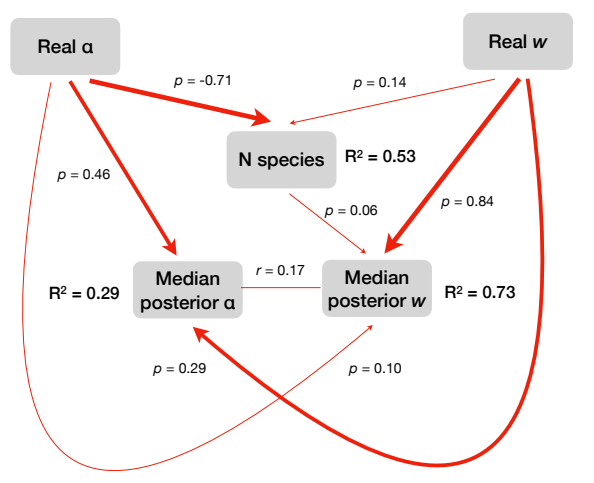

B)

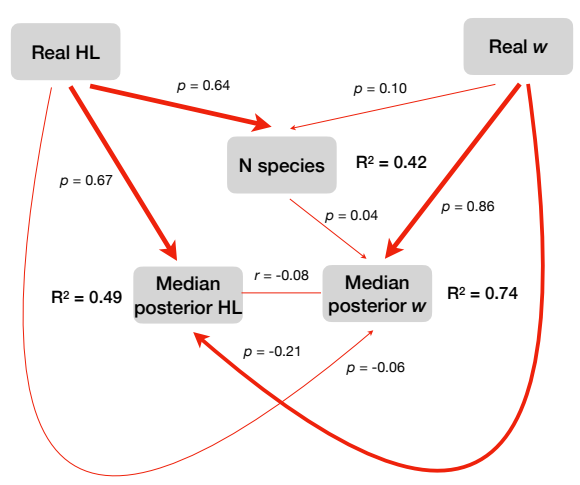

D)

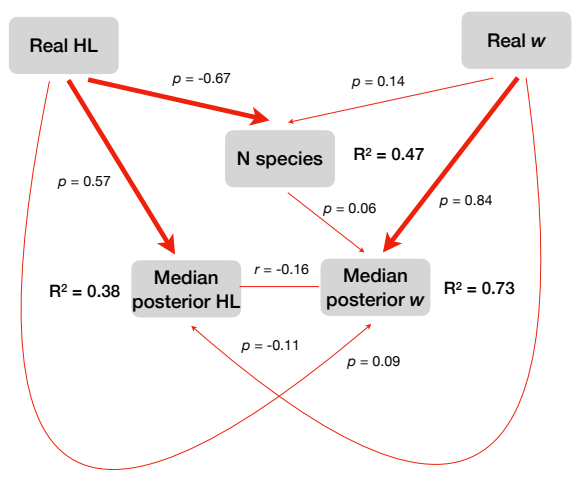

Figure 5: Causal models relating real OU's $\alpha$ or half-life, real $w$ slope and number of species in 1,000 simulated metacommunities on the median of the posterior distribution of OU's $\alpha$ of half-life and $w$ slope estimated using uniform $(A, B)$ or half-life derived $(C, D)$ OU's $\alpha$ prior. Causal relationships were estimated using ordinary least squares. Half-life and $w$ slopes were log-transformed prior to analysis. Validity of overall models was tested using d-separation tests (Shipley 2000). A) OU's $\alpha$ model, uniform $\alpha$ prior (Fisher's $\mathrm{C}=1.59, P_{\mathrm{df}=2}=0.45$ ). $B$ ) Half-life model, uniform $\alpha$ prior (Fisher's $\mathrm{C}=0.63, P_{\mathrm{df}=2}=$ 0.73). C) OU's $\alpha$ model, half-life $\alpha$ prior (Fisher's $C=4.13, P_{\mathrm{df}=2}=0.13$ ). $D$ ) Half-life model, half-life $\alpha$ prior (Fisher's $\mathrm{C}=4.64, P_{\mathrm{df}=2}=0.10$ ). Arrow width indicates the strength of the respective causal relationship.

\section{Phyllostomid bats}

ABC analysis of phyllostomid bats generated a prior distribution of OU's $\alpha$ and $w$ slope parameters containing 16,040 simulated metacommunities. Both prior and posterior distribution of parameters are shown in figure 6. The posterior distribution of OU's $\alpha$ (fig. $6 A$ ) indicated a density peak at $\alpha=0.05$, which means a niche half-life $\left(\mathrm{t}_{1 / 2}=\right) 13.9$ Myr, or $41 \%$ of the total timespan of the phylogenetic tree of phyllostomids (34 Myr).

That is to say that the distribution of entropy across the temperature seasonality gradient showed high phylogenetic signal.

Moreover, the analysis estimated a peak density for $w$ slope $=58.52$. The minimum spanning tree computed based on the geographic coordinates of sites indicated that the maximum distance necessary to connect all cells in the map was 263 kilometers. Based on eq. 3, at such distance the probability of a species to disperse from 
708 a site to another was very high $\left(\delta_{l k}>0.96\right.$, see the subplot within fig. $\left.6 B\right)$; the

709 probability $\delta_{l k}$ only decreases to 0.5 at distances $\cong 1,000$ kilometers.

$710 \quad$ Figure $7 A$ shows the observed entropy distribution of bat assemblages across the

711 Neotropic, while figure $7 B$ provides mean estimates of entropy computed from those

712 simulations whose OU's $\alpha$ and $w$ slope parameters fell within the boundaries of the

713 most representative HPD computed for both parameters. As we can see, ABC algorithm

714 captured most entropy variation across sites. In figure $7 C$, we plotted the residuals of a

715 cubic model (subplot within fig. 7C) relating the observed entropy of sites (y-axis) to

716 ABC-estimated entropy ( $\mathrm{x}$-axis). As we can see, although there is a strong association

717 between entropies, it is not quite linear, especially at lower and upper limits of entropy

718 values. Interestingly, the residual plot indicates that in the southernmost regions of the

719 Neotropic, where entropy values were lower, the simulation framework either under or

720 overestimated the entropy of bat assemblages (fig. 7C). 

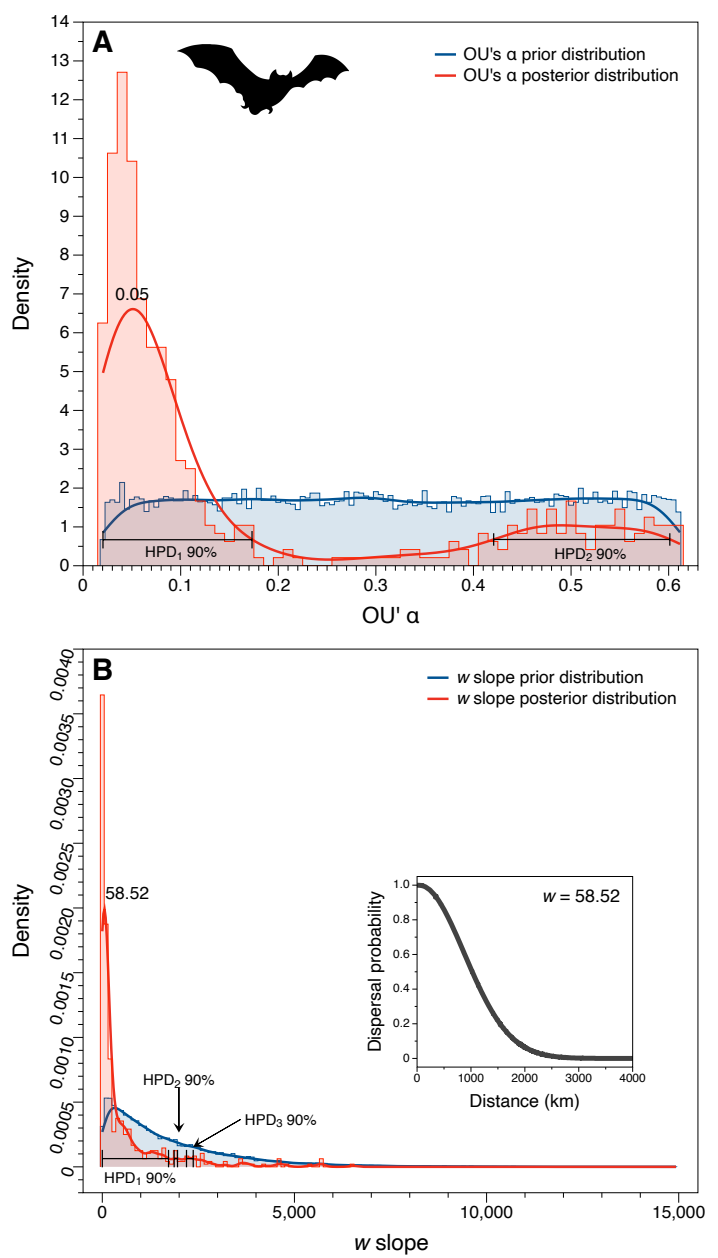

$723 \quad$ Figure 6: Histograms and density plots of the posterior distribution of $(A)$ OU's $\alpha$ and $(B) w$ slope parameters underlying species diversity of phyllostomid bats along a gradient in temperature seasonality in the Neotropic. Parameters were estimated using ABC analysis implemented in mcfly package. Prior sample size: 16,040 simulations. Posterior sample size: 480 simulations. Only the most representative highest posterior density intervals (HPD $190 \%$ ) were interpreted. 

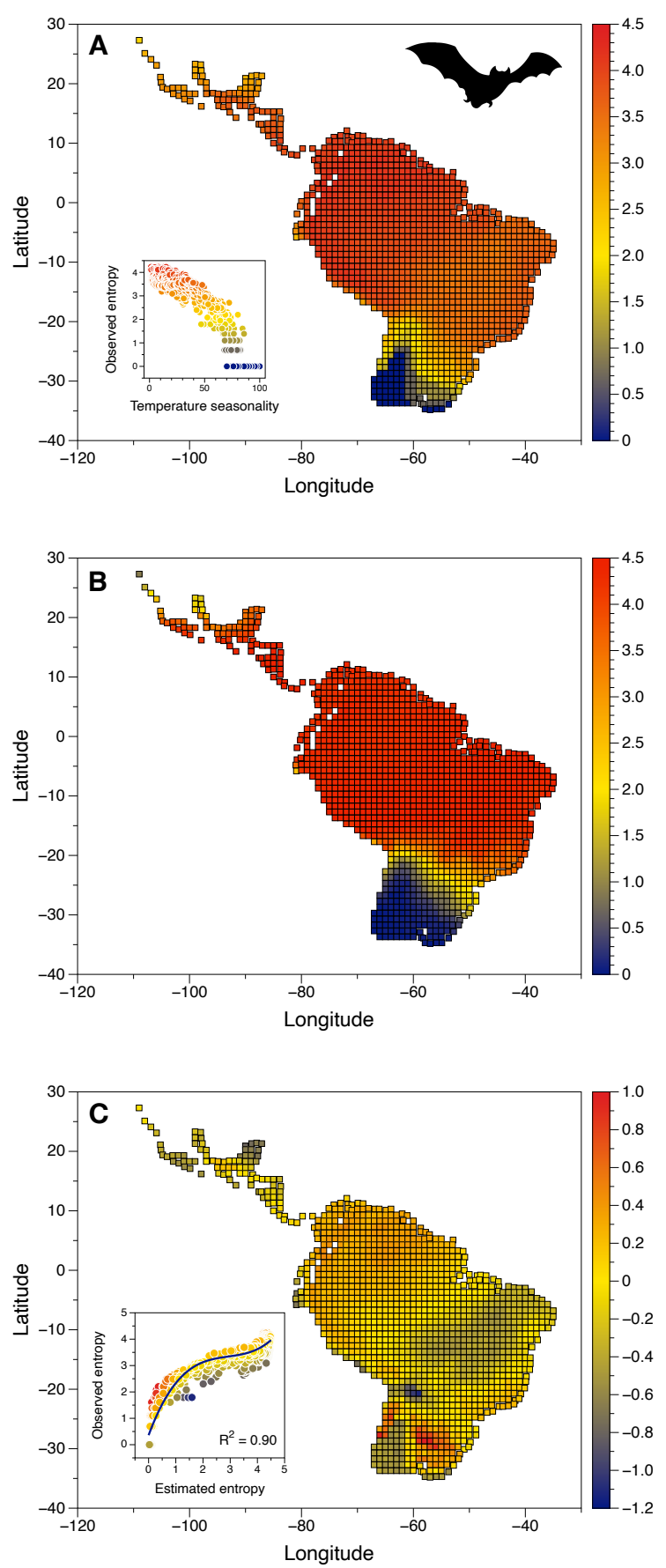

Figure 7: Species diversity gradients of phyllostomid bats across the Neotropic. (A) Observed entropy. The subplot within shows strong negative correlation between entropy and temperature seasonality, with highest entropy values associated with lower temperature seasonality. $(B)$ mean entropy estimated using $\mathrm{ABC}$ analysis. Mean values were computed for simulations with OU's $\alpha$ and $w$ slopes falling within the boundaries of the most representative HPD interval (see fig. 6) computed for the corresponding posterior distribution of each estimated parameter. (C) Residual values of the cubic model relating estimated ( $\mathrm{x}-$ axis) and observed entropy (y-axis) (see subplot within the main plot). 


\section{Discussion}

740 The framework proposed in this study and implemented in the package $m c f l y$

741 successfully enabled us to infer the most plausible scenario of niche evolution

742 determining biological diversity gradients across spatially-structured environmental

743 gradients. We demonstrated that our model-based simulation approach, coupled to

744 rejection-based Approximate Bayesian Computation (ABC) algorithm (Beaumont 2010;

745 Csilléry et al. 2010), allows to estimate (i) the strength of adaptation rate along the niche

746 evolution of species from a monophyletic lineage and distributed across

747 metacommunities; and (ii) the role played by dispersal limitation on the distribution of

748 biological diversity across space. Considering the initial aims of community

749 phylogenetics proposed by Webb et al. (2002), our approach provides meaningful

750 estimates of the role played by phylogenetic relationships on species assembly patterns,

751 and helps to reconcile niche and neutral processes, a topic heatedly debated over the last

752 decades (Vellend 2016, and references therein). Previous studies have already pointed

753 out the need of integrating niche evolution to diversity gradients (Cadotte et al. 2017).

754 This has been explored with correlative approaches relating phylogenetic and trait

755 diversity (Tucker et al. 2018) or, indirectly, by estimating phylogenetic signal in one or

756 more traits used to compute functional diversity (Muscarella et al. 2016). However, we

757 demonstrated it is possible to link niche evolution models and species diversity

758 gradients without assuming a priori phenotypic traits to define species niches. This

759 releases the need of assuming a given trait (or group of traits) as the most appropriate

760 for assessing niche-environment associations (why not any other[s]?). An advantage of

761 our approach is that it allows the evaluation of datasets for which a complete

762 characterization of niche dimensions is currently not available. Thus, it may fill an

763 important gap in the knowledge on the relationship between phylogenetic relationships

764 among species and ecological diversity.

765 The analyses performed using simulated datasets demonstrated that the $\mathrm{ABC}$

766 approach implemented in $m c f l y$ is suitable to estimate the adaptation rate of species

767 niche positions and the degree of dispersal limitation across the assemblages in a

768 metacommunity based on species diversity variation across environmental gradients.

769 Nonetheless, the analytical framework showed to be sensitive to the mode of the prior

770 OU's $\alpha$ distribution (Roos et al. 2015). Therefore, we recommend the use of the uniform

771 distribution of OU's $\alpha$ to avoid biased posterior distributions of OU's $\alpha$ parameter.

772 Causal models detected solid links between real and estimated niche parameters (and 
773 for the dispersal parameter - $w$ slope), either where OU's $\alpha$ or niche half-life was

774 considered. Thus, even though a formally defined function relating biological diversity

775 to evolutionary models of niche evolution is not available to date, the $\mathrm{ABC}$ approach

776 implemented in the package $m c f l y$ proved to be a useful way to estimate the imprint of

777 niche evolution on diversity gradients (Cadotte et al. 2017). Although evolutionary

778 analyses usually suffer from incomplete datasets (FitzJohn et al. 2009; Nee et al. 1994;

779 Pybus and Harvey 2000), our framework demonstrated to be robust even for

780 metacommunities showing incomplete phylogenetic data (simulated metacommunities

781 contained at least $40 \%$ of total phylogenetic pool), which is quite common in ecological

782 and biogeographic data. Yet, some caution may be necessary where regional assembly

783 contains only a small fraction of the species within the phylogenetic tree.

784 Phyllostomid bats showed strong dependency on niche evolution towards an

785 optimum of low temperature seasonality across Neotropical assemblages, corroborating

786 results obtained by Stevens (2011). Phyllostomids have high dispersal capacity up to

787 distances of $c a .1000 \mathrm{~km}$, which is in conformity with the large geographic range of

788 most of its species (Willig et al. 2003). Moreover, the estimated OU' $\alpha$ of phyllostomid's

789 niche positions was low $(\alpha=0.05)$, which confers a relatively high half-life to niche

790 positions of species (13.9 Myr). Those results suggest that those bats preferably track

791 habitats offering niche conditions close to their optimum over long distances, instead of

792 quickly adapting their niches towards an optimum condition (see also Villalobos et al.

793 2013). That strategy was already hypothesized to explain plant distribution (Donoghue

794 2008). This finding may explain evidence showing that closely related phyllostomid

795 species tend to live in geographic proximity (Villalobos et al. 2014).

796 In general, estimated entropy closely mirrored observed richness in bat

797 assemblages, although not quite linearly, as revealed by the model relating observed to

798 estimated entropy. For instance, at higher observed entropy levels in Amazonia region,

799 simulations tended to slightly underestimate entropy. Certainly, another relevant niche

800 factors might enable diversity levels to be higher than those expected by climatic niche

801 alone. Vertical stratification in tropical rainforests, associated with seasonal floods

802 affecting nutrient and roost availability, is a well-known driver of local phyllostomid

803 diversity in Amazonian forests (Bernard 2001; Pereira et al. 2010; Pereira et al. 2009).

804 Some other remarkable deviation patterns were observed in two regions: (1) Southern

805 Atlantic forests, where entropy was strongly underestimated. Here, phyllostomids are a

806 small subsample of the diversity of the family, as may be inferred from the IUCN 
807 distribution maps (Rojas et al. 2018a), and are mostly represented by generalist

808 frugivores who present higher speciation rates than phyllostomids of other feeding

809 guilds (Rojas et al. 2018b). Such generalist diet would allow them to cope with the

810 unpredictability of food resources in the subtropical regions. (2) The "dry" diagonal of

811 South America, where entropy was in general overestimated. Neotropical rainforests

812 and savannas share a significant part of the generalist frugivore assemblage, but the

813 absence of dense forests in the more open physiognomies negatively affects the

814 occurrence of several frugivorous phyllostomids (Stevens 2006), particularly those

815 specialized in canopy fruits. Therefore, the phyllostomid assemblages of the South

816 American "dry" diagonal become more represented by obligatory, and specialized,

817 nectarivores, which show lower rates of speciation than those observed for the

818 generalist herbivore lineages (Rojas et al. 2018b); this might explain entropy

819 overestimation. In all cases, a single optimum niche condition might fail to account for

820 other relevant variables such as vegetation types. Indeed, the current version of $m c f l y$

821 has this limitation, which should be fixed in further developments of the analytical

822 framework. Yet, in its current version, $m c f l y$ allows for multiple conditions to be

823 included as a principal component, and users may thereby incorporate climatic and

824 landscape data to the analysis. Finally, mismatches between observed and estimated

825 entropy in some areas may be, to some extent, due to inconsistencies in IUCN maps.

826 The framework proposed here shed light on the link between niche evolution

827 and the distribution of biological diversity, and thereby improved our understanding of

828 evolutionary imprints on ecological patterns. Yet, we believe that the main strength of

829 this new approach is to open avenues of investigation that may help solving the eco-

830 evolutionary puzzle. For instance, multiple niche optima, possibly derived from

831 different niche conditions, and affecting differentially distinct clades, might provide

832 more realistic scenarios than the single optimum scenario developed here. Further, our

833 framework deals with evolutionary imprints on ecological patterns, but it does not allow

834 evaluating how higher ecological mechanisms (sensu Vellend 2016) may ultimately

835 affect evolutionary dynamics (Weber et al. 2017). Further developments of the

836 framework proposed here should help filling these (and other) gaps in near future. This

837 tale is to be continued. 
839 LD, MVC, MJRP and JAFDF research activities have been supported by CNPq

840 Productivity Fellowship (grants 307527/2018-2, 306590/2018-2, 311297/2018-8 and

841 301799/2016-4, respectively). LD, GN, MVC and JAFDF research have been

842 conducted in the context of the National Institutes for Science and Technology (INCT)

843 in Ecology, Evolution and Biodiversity Conservation, supported by MCTIC/CNPq

844 (proc. 465610/2014-5) and FAPEG (proc. 201810267000023). The PhyloPic

845 (http://phylopic.org) image of a phyllostomid bat used in figures 6 and 7 was created by

846 Melissa Ingala, and its use was authorized under the license available at

847 https://creativecommons.org/licenses/by/3.0/. Figure 1 was brilliantly designed by Clara

848 Heinrich (linkedin.com/in/clara-heinrich-4bb3a3112).

849 A ciência brasileira resiste.

\section{References}

Ackerly, D. D. 2003. Community assembly, niche conservatism, and adaptive evolution in changing environments. International Journal of Plant Sciences 164:S165-S184.

Anand, M., and L. Orlóci. 1996. Complexity in plant communities: the notion and quantification. Journal of Theoretical Biology 179:179-186.

Baker, R. J., O. R. P. Bininda-Emonds, H. Mantilla-Meluk, C. A. Porter, and R. A. V. D. Bussche. 2012. Molecular time scale of diversification of feeding strategy and morphology in NewWorld Leaf-Nosed Bats (Phyllostomidae): a phylogenetic perspective, Pages 385-409. Evolutionary history of bats. Cambridge, U.K., Cambridge Univ. Press.

860 Beaumont, M. A. 2010. Approximate Bayesian computation in evolution and ecology. Annual review of ecology, evolution, and systematics 41:379-406.

862 Beaumont, M. A., W. Zhang, and D. J. Balding. 2002. Approximate Bayesian computation in population genetics. Genetics 162:2025-2035.

864 Bell, G. 2000. The distribution of abundance in neutral communities. The American Naturalist 155:606-617.

866 Bernard, E. 2001. Vertical stratification of bat communities in primary forests of Central Amazon, Brazil. Journal of Tropical Ecology 17:115-126.

868 Blomberg, S. P., and T. Garland. 2002. Tempo and mode in evolution: phylogenetic inertia, adaptation and comparative methods. Journal of Evolutionary Biology 15:899-910.

Borcard, D., and P. Legendre. 2002. All-scale spatial analysis of ecological data by means of principal coordinates of neighbour matrices. Ecological Modelling 153:51-68.

872 Butler, M., and A. King. 2004. Phylogenetic comparative analysis: A modeling approach for adaptive evolution. The American Naturalist 164:683-695. 
874 Cadotte, M. W., R. Dinnage, and D. Tilman. 2012. Phylogenetic diversity promotes ecosystem stability. Ecology 93:S223-S233.

876 Cadotte, M. W., T. Jonathan Davies, and P. R. Peres-Neto. 2017. Why phylogenies do not always predict ecological differences. Ecological Monographs 87:535-551.

Cavender-Bares, J., K. H. Kozak, P. V. A. Fine, and S. W. Kembel. 2009. The merging of community ecology and phylogenetic biology. Ecology Letters 12:693-715.

Cooper, N., W. Jetz, and R. P. Freckleton. 2010. Phylogenetic comparative approaches for studying niche conservatism. Journal of Evolutionary Biology 23:2529-2539.

Cooper, N., G. H. Thomas, C. Venditti, A. Meade, and R. P. Freckleton. 2016. A cautionary note on the use of Ornstein Uhlenbeck models in macroevolutionary studies. Biological Journal of the Linnean Society 118:64-77.

Crisp, M. D., and L. G. Cook. 2005. Do early branching lineages signify ancestral traits? Trends in Ecology \& Evolution 20:122-128.

Csilléry, K., M. G. Blum, O. E. Gaggiotti, and O. François. 2010. Approximate Bayesian computation $(\mathrm{ABC})$ in practice. Trends in ecology \& evolution 25:410-418.

Del Monte-Luna, P., B. W. Brook, M. J. Zetina-Rejón, and V. H. Cruz-Escalona. 2004. The carrying capacity of ecosystems. Global ecology and biogeography 13:485-495.

Donoghue, M. J. 2008. A phylogenetic perspective on the distribution of plant diversity. Proceedings of the National Academy of Sciences 105:11549-11555.

Dray, S., and P. Legendre. 2008. Testing the species traits-environment relationships: the fourth-corner problem revisited. Ecology 89:3400-3412.

Duarte, L. D. S., V. J. Debastiani, M. B. Carlucci, and J. A. F. Diniz-Filho. 2018. Analyzing community-weighted trait means across environmental gradients: should phylogeny stay or should it go? Ecology 99:385-398.

898 Duarte, L. d. S., R. E. Machado, S. M. Hartz, and V. D. Pillar. 2006. What saplings can tell us about forest expansion over natural grasslands. Journal of Vegetation Science 17:799808.

Duarte, L. D. S., P. V. Prieto, and V. D. Pillar. 2012. Assessing spatial and environmental

903 Elton, C. 1927, Animal Ecology. London, Sedgwick and Jackson.

904 Enquist, B. J., J. Norberg, S. P. Bonser, C. Violle, C. T. Webb, A. Henderson, L. L. Sloat et al. 2015. Scaling from Traits to Ecosystems: Developing a General Trait Driver Theory via Integrating Trait-Based and Metabolic Scaling Theories. . Advances in Ecological

908 Felsenstein, J. 1985. Phylogenies and the comparative method. American Naturalist 125:1-15. 
Fick, S. E., and R. J. Hijmans. 2017. WorldClim 2: new 1-km spatial resolution climate surfaces for global land areas. International Journal of Climatology.

FitzJohn, R. G., W. P. Maddison, and S. P. Otto. 2009. Estimating trait-dependent speciation and extinction rates from incompletely resolved phylogenies. Systematic biology 58:595-611.

914 Fleming, T. H., L. D. Dávalos, and M. A. R. Mello. 2020, Phyllostomidae bats: a unique

915 mammalian radiation. Chicago, The University of Chicago Press

916 Garnier, E., M.-L. Navas, and K. Grigulis. 2016, Plant functional diversity: organism traits,

917 community structure, and ecosystem properties., Oxford University Press.

918 Gerhold, P., J. F. Cahill, M. Winter, I. V. Bartish, and A. Prinzing. 2015. Phylogenetic patterns are not proxies of community assembly mechanisms (they are far better). Functional Ecology 29:600-614.

Gerhold, P., M. B. Carlucci, Ş. Procheş, and A. Prinzing. 2018. The deep past controls the phylogenetic structure of present, local communities. Annual Review of Ecology, Evolution, and Systematics 49:477-497.

Godoy, O., N. J. B. Kraft, and J. M. Levine. 2014. Phylogenetic relatedness and the determinants of competitive outcomes. Ecology Letters 17:836-844.

Gould, S. J., and R. C. Lewontin. 1979. The spandrels of San Marco and the Panglossian paradigm: a critique of the adaptationist programme. Proceedings of the Royal Society of London. Series B. Biological Sciences 205:581-598.

Gravel, D., C. D. Canham, M. Beaudet, and C. Messier. 2006. Reconciling niche and neutrality: the continuum hypothesis. Ecology Letters 9:399-409.

Hansen, T. F. 1997. Stabilizing selection and the comparative analysis of adaptation. Evolution 51:1341-1351.

Hansen, T. F., and E. P. Martins. 1996. Translating between microevolutionary process and macroevolutionary patterns: the correlation structure of interspecific data. Evolution 50:1404-1417.

Hansen, T. F., J. Pienaar, and S. H. Orzack. 2008. A comparative method for studying adaptation to a randomly evolving environment. Evolution: International Journal of Organic Evolution 62:1965-1977.

Harmon, L. J., J. T. Weir, C. D. Brock, R. E. Glor, and W. Challenger. 2008. GEIGER: investigating evolutionary radiations. Bioinformatics 24:129-131.

Harvey, P. H., and M. D. Pagel. 1991, The comparative method in evolutionary biology.

943 Hubbell, S. P. 2001, The Unified Neutral Theory of Biodiversity and Biogeography. Princeton, 
Hutchinson, G. E. 1957. Concluding remarks. Cold Spring Harbor Symposia on Quantitative Biology 22:415-427.

947 Hutchinson, G. E. 1959. Homage to Santa Rosalia or why are there so many kinds of animals?

948 The American Naturalist 93:145-159.

949 IUCN. 2020. The IUCN Red List of Threatened Species. Version 6.2.

950 Kraft, N. J. B., P. B. Adler, O. Godoy, E. James, S. Fuller, and J. M. Levine. 2015. Community assembly, coexistence, and the environmental filtering metaphor. Functional Ecology 29:592-599.

Lefcheck, J. S. 2016. piecewiseSEM: Piecewise structural equation modelling in r for ecology, evolution, and systematics. Methods in Ecology and Evolution 7:573-579.

956 Leibold, M. A., M. Holyoak, N. Mouquet, P. Amarasekare, J. M. Chase, M. F. Hoopes, R. D. Holt et al. 2004. The metacommunity concept: a framework for multi-scale community ecology. Ecology Letters 7:601-613.

Levine, J. M., and J. HilleRisLambers. 2009. The importance of niches for the maintenance of species diversity. Nature 461:254-257.

961 Losos, J. B. 2008. Phylogenetic niche conservatism, phylogenetic signal and the relationship between phylogenetic relatedness and ecological similarity among species. Ecology Letters 11:995-1003.

964

Loyola, R. D., P. Lemes, F. T. Brum, D. B. Provete, and L. D. S. Duarte. 2013. Clade-specific 965 consequences of climate change to amphibians in Atlantic Forest protected areas. Ecography 37:65-72.

MacArthur, R., and R. Levins. 1967. The limiting similarity, convergence, and divergence of coexisting species. The American Naturalist 101:377-385.

MacArthur, R. H. 1972, Geographical Ecology: Patterns in the Distribution of Species. Princeton, Princeton University Press.

Maestri, R., and L. Duarte. 2020. Evolutionary imprints on species distribution patterns across the Neotropics, Pages 103-119 Neotropical Diversification: Patterns and Processes, Springer.

974 Mayfield, M. M., and J. M. Levine. 2010. Opposing effects of competitive exclusion on the phylogenetic structure of communities. Ecology Letters 13:1085-1093. comprehensive model. Vegetatio 71:145-156.

979 Monteiro, L. R., and M. R. Nogueira. 2011. Evolutionary patterns and processes in the radiation of phyllostomid bats. BMC Evolutionary Biology 11:1. 
Morrone, J. J. 2014. Biogeographical regionalisation of the Netropical region. Zootaxa 3782:001-110

Mouquet, N., V. Devictor, C. N. Meynard, F. Munoz, L.-F. Bersier, J. Chave, P. Couteron et al. 2012. Ecophylogenetics: advances and perspectives. Biological Reviews 87:769-785.

Münkemüller, T., F. C. Boucher, W. Thuiller, and S. Lavergne. 2015. Phylogenetic niche conservatism - common pitfalls and ways forward. Functional Ecology 29:627-639.

Muscarella, R., M. Uriarte, T. M. Aide, D. L. Erickson, J. Forero-Montaña, W. J. Kress, N. G. Swenson et al. 2016. Functional convergence and phylogenetic divergence during secondary succession of subtropical wet forests in Puerto Rico. Journal of Vegetation Science 27:283-294.

Nakamura, G., L. O. Gonçalves, and L. d. S. Duarte. 2020. Revisiting the dimensionality of biological diversity. Ecography 43:539-548.

Nathan, R., and H. C. Muller-Landau. 2000. Spatial patterns of seed dispersal, their

996

997 determinants and consequences for recruitment. Trends in Ecology \& Evolution 15:278285.

Nee, S., R. M. May, and P. H. Harvey. 1994. The reconstructed evolutionary process. Philosophical Transactions of the Royal Society of London. Series B: Biological Sciences 344:305-311.

Nosil, P. 2012, Ecological speciation, Oxford University Press.

Paine, C. T., A. Deasey, and A. B. Duthie. 2018. Towards the general mechanistic prediction of community dynamics. Functional ecology 32:1681-1692.

Paradis, E., and K. Schliep. 2019. ape 5.0: an environment for modern phylogenetics and evolutionary analyses in R. Bioinformatics 35:526-528.

Pereira, M. J. R., J. T. Marques, and J. M. Palmeirim. 2010. Vertical stratification of bat assemblages in flooded and unflooded Amazonian forests. Current Zoology 56:469478.

Pereira, M. J. R., J. T. Marques, J. Santana, C. D. Santos, J. Valsecchi, H. L. De Queiroz, P. Beja et al. 2009. Structuring of Amazonian bat assemblages: the roles of flooding patterns and floodwater nutrient load. Journal of Animal Ecology 78:1163-1171.

Pereira, M. J. R., and J. M. Palmeirim. 2013. Latitudinal diversity gradients in New World Bats: are they a consequence of niche conservatism? Plos One 8:e69245.

Peres-Neto, P. R., S. Dray, and C. J. F. t. Braak. 2017. Linking trait variation to the environment: critical issues with community-weighted mean correlation resolved by the fourth-corner approach. Ecography 40:806-816.

Peres-Neto, P. R., M. A. Leibold, and S. Dray. 2012. Assessing the effects of spatial contingency and environmental filtering on metacommunity phylogenetics. Ecology 93:S14-S30. 
1018 Pybus, O. G., and P. H. Harvey. 2000. Testing macro-evolutionary models using incomplete molecular phylogenies. Proceedings of the Royal Society of London. Series B: Biological Sciences 267:2267-2272.

Pyron, R. A., G. C. Costa, M. A. Patten, and F. T. Burbrink. 2015. Phylogenetic niche conservatism and the evolutionary basis of ecological speciation. Biological Reviews 90:1248-1262.

Rényi, A. 1961, On measures of entropy and information Proceedings of the Fourth Berkeley Symposium on Mathematical Statistics and Probability, Volume 1: Contributions to the Theory of Statistics.

Revell, L. J., L. J. Harmon, and D. C. Collar. 2008. Phylogenetic signal, evolutionary process, and rate. Systematic Biology 57:591-601.

Ricklefs, R. E., and D. Schluter. 1993. Species diversity in ecological communities: historical and geographical perspectives.

Ricotta, C. 2005. Through the Jungle of Biological Diversity. Acta Biotheoretica 53:29-38.

Rojas, D., M. Moreira, M. J. Ramos Pereira, C. Fonseca, and L. M. Dávalos. 2018a. Updated distribution maps for neotropical bats in the superfamily Noctilionoidea. Ecology 99:2131-2131.

Rojas, D., M. J. Ramos Pereira, C. Fonseca, and L. M. Dávalos. 2018b. Eating down the food chain: generalism is not an evolutionary dead end for herbivores. Ecology Letters 21:402-410.

Roos, M., T. G. Martins, L. Held, and H. Rue. 2015. Sensitivity Analysis for Bayesian Hierarchical Models. Bayesian Analysis 10:321-349, 329.

Rossoni, D. M., B. M. A. Costa, N. P. Giannini, and G. Marroig. 2019. A multiple peak adaptive landscape based on feeding strategies and roosting ecology shaped the evolution of cranial covariance structure and morphological differentiation in phyllostomid bats. Evolution 73:961-981. ecological niche breadth. Annual Review of Ecology, Evolution, and Systematics 48.

Shi, J. J., and D. L. Rabosky. 2015. Speciation dynamics during the global radiation of extant bats. Evolution 69:1528-1545.

Shipley, B. 2000, Cause and correlation in biology: a user's guide to path analysis, structural equations and causal inference, Cambridge Unversity Press.

- 2010, From plant traits to vegetation structure: chance and selection in the assembly of ecological communities, Cambridge University Press.

Simmons, N. B., and A. L. Cirranello. 2020. Bat Species of the World: A taxonomic and geographic database. 
Sokol, E. R., B. L. Brown, and J. Barrett. 2017. A simulation-based approach to understand how

1055

1056

1057

1058

1059

1060

1061

1062

1063

1064

1065

1066

1067

1068

1069

1070

1071

1072

1073

1074

1075

1076

1077

1078

1079

1080

1081

1082

1083

1084

1085

1086

1087

1088

1089

metacommunity characteristics influence emergent biodiversity patterns. Oikos 126:723-737.

Sokol, E. R., B. L. Brown, C. C. Carey, B. M. Tornwall, C. M. Swan, and J. E. Barrett. 2015. Linking management to biodiversity in built ponds using metacommunity simulations. Ecological Modelling 296:36-45.

Stevens, R. D. 2006. Historical processes enhance patterns of diversity along latitudinal gradients. Proceedings. Biological sciences 273:2283-2289.

- 2011. Relative effects of time for speciation and tropical niche conservatism on the latitudinal diversity gradient of phyllostomid bats. Proceedings of the Royal Society B: Biological Sciences 278:2528-2536.

Swenson, N. G. 2019, Phylogenetic Ecology: A History, Critique, and Remodeling, University of Chicago Press.

Tucker, C. M., T. J. Davies, M. W. Cadotte, and W. D. Pearse. 2018. On the relationship between phylogenetic diversity and trait diversity. Ecology 99:1473-1479.

Turkkan, N., and T. Pham-Gia. 1993. Computation of the highest posterior density interval in bayesian analysis. Journal of Statistical Computation and Simulation 44:243-250.

Vellend, M. 2016, The Theory of Ecological Communities, Princeton University Press.

Villalobos, F., and H. T. Arita. 2010. The diversity field of New World leaf-nosed bats (Phyllostomidae). Global Ecology and Biogeography 19:200-211.

Villalobos, F., A. Lira-Noriega, J. Soberón, and H. T. Arita. 2014. Co-diversity and codistribution in phyllostomid bats: Evaluating the relative roles of climate and niche conservatism. Basic and Applied Ecology 15:85-91.

Villalobos, F., T. F. Rangel, and J. A. F. Diniz-Filho. 2013. Phylogenetic fields of species: cross-species patterns of phylogenetic structure and geographical coexistence. Proceedings of the Royal Society B: Biological Sciences 280.

Violle, C., B. J. Enquist, B. J. McGill, L. Jiang, C. H. Albert, C. Hulshof, V. Jung et al. 2012. The return of the variance: intraspecific variability in community ecology. Trends in Ecology \& Evolution 27:244-252.

Violle, C., M.-L. Navas, D. Vile, E. Kazakou, C. Fortunel, I. Hummel, and E. Garnier. 2007. Let the concept of trait be functional! . Oikos 116:882-892.

Webb, C. O., D. D. Ackerly, M. A. McPeek, and M. J. Donoghue. 2002. Phylogenies and community ecology. Annual Review of Ecology and Systematics 33:475-505.

Weber, M. G., C. E. Wagner, R. J. Best, L. J. Harmon, and B. Matthews. 2017. Evolution in a community context: on integrating ecological interactions and macroevolution. Trends in Ecology \& Evolution 32:291-304. 
1090 Wiens, J. J., and C. H. Graham. 2005. Niche conservatism: integrating evolution, ecology, and conservation biology. Annual Review of Ecology, Evolution, and Systematics 36:519539.

1093 Willig, M. R., B. D. Patterson, and R. D. Stevens. 2003. Patterns of range size, richness, and

1094 body size in the Chiroptera, Pages 580-621 in T. H. Kunz, and M. B. Fenton, eds. Bat Ecology Chicago, Univ. Chicago Press.

1096 
1097 Figure 1: Schematic representation of $m c f l y$, an Approximate Bayesian Computation

1098 framework developed to estimate the posterior distribution of adaptation rate (OU's $\alpha$ )

1099 and dispersal kernel slope $(w)$ parameters driving species diversity gradients along an

1100 environmental gradient. 1. The real metacommunity provides the empirical measures of

1101 species diversity, the real summary statistic $(R)$. 2. Environment is a dimension of

1102 species niches that provides the optimum niche $(\theta)$, variance $(\sigma)$ and root niche position

1103 parameters necessary to simulate species niche based on the phylogeny, according to

1104 Ornstein-Uhlenbeck evolutionary model. We also define niche breadth in the simulation

1105 based on environment. 3. A prior distribution of OU's $\alpha$ is used to simulate species

1106 niche positions based on environment parameters and phylogeny. 4. Spatial coordinates

1107 of $k$ sites are used to define the prior distribution of slope $w$; together with immigration

1108 rate $(m)$, number of individuals at $k$ sites $\left(J_{L_{-} k}\right)$ and the total number of individuals in $M$

$1109\left(J_{M}\right)$ provide the neutral parameters of simulations. 5. Metacommunities are simulated

1110 based on niche and neutral parameters (Simulator). 6. Each metacommunity simulation

1111 provides species diversity measures (Summarizer) taken as the simulated summary

1112 statistics $(S)$. 7. Simulated diversities $(S)$ are classified based on a pre-defined minimum

1113 correlation threshold (tolerance) to the observed diversity $(R)$. Every $S$ object not

1114 discarded (Reject) is then scanned to record its OU's $\alpha$ and slope $w$ to provide a

1115 posterior distribution of adaptation rate $(\alpha)$ and dispersal limitation $(w)$, respectively.

1116 Figure 2: Relationship between Blomberg's K statistic and the adaptation rate

1117 parameter of Ornstein-Uhlenbeck model (OU's $\alpha$ ) for simulated niche positions

1118 computed for 1,000 sets of 50 species each (black circles). Gray areas indicate lower

1119 and upper credibility intervals (95\%) for Blomberg's K and OU's $\alpha$ values estimated

1120 from white noise vectors $(N[0,1])$.

1121 Figure 3: Causal models relating real OU's $\alpha$ or half-life, real $w$ slope, OU's $\alpha$ prior

1122 mode and number of species in 2,000 simulated metacommunities on the median of the

1123 posterior distribution of OU's $\alpha$ of half-life and $w$ slope. Causal relationships were

1124 estimated using ordinary least squares. Half-life and $w$ slopes were log-transformed

1125 prior to analysis. Validity of overall models was tested using d-separation tests (Shipley

1126 2000). $A$ ) OU's $\alpha$ model (Fisher's $\mathrm{C}=2.67, P_{\mathrm{df}=4}=0.62$ ). $B$ ) Half-life model (Fisher's C

$\left.1127=1.18, P_{\mathrm{df}=4}=0.88\right)$. Arrow width indicates the strength of the respective causal

1128 relationship. 
1129 Figure 4: Bivariate relationships between real niche (OU's $\alpha$ or half-life) or dispersal

1130 ( $w$ slope) parameters of simulated metacommunities and the corresponding median of

1131 the posterior distribution of OU's $\alpha$, half-life and $w$ slope. $A, C, E$ ) Uniform OU's $\alpha$

1132 prior. $B, D, F)$ Half-life derived OU's $\alpha$ prior. Dashed lines delimit confidence intervals

$1133(95 \%)$ of predictions.

1134 Figure 5: Causal models relating real OU's $\alpha$ or half-life, real $w$ slope and number of

1135 species in 1,000 simulated metacommunities on the median of the posterior distribution

1136 of OU's $\alpha$ of half-life and $w$ slope estimated using uniform $(\mathrm{a}, \mathrm{b})$ or half-life derived (c,

1137 d) OU's $\alpha$ prior. Causal relationships were estimated using ordinary least squares. Half-

1138 life and $w$ slopes were log-transformed prior to analysis. Validity of overall models was

1139 tested using d-separation tests (Shipley 2000). A) OU's $\alpha$ model, uniform $\alpha$ prior

1140 (Fisher's $\left.\mathrm{C}=1.59, P_{\mathrm{df}=2}=0.45\right) . B$ ) Half-life model, uniform $\alpha$ prior $($ Fisher's $\mathrm{C}=0.63$,

$\left.1141 P_{\mathrm{df}=2}=0.73\right) . C$ ) OU's $\alpha$ model, half-life $\alpha$ prior (Fisher's C=4.13, $\left.P_{\mathrm{df}=2}=0.13\right) . D$ )

1142 Half-life model, half-life $\alpha$ prior (Fisher's $\mathrm{C}=4.64, P_{\mathrm{df}=2}=0.10$ ). Arrow width indicates

1143 the strength of the respective causal relationship.

1144 Figure 6: Histograms and density plots of the posterior distribution of (a) OU's $\alpha$ and

1145 (b) $w$ slope parameters underlying species diversity of phyllostomid bats along a

1146 gradient in temperature seasonality in the Neotropic. Parameters were estimated using

1147 ABC analysis implemented in $m c f l y$ package. Prior sample size: 16,040 simulations.

1148 Posterior sample size: 480 simulations. Only the most representative highest posterior

1149 density intervals $\left(\mathrm{HPD}_{1} 90 \%\right)$ were interpreted.

1150 Figure 7: Species diversity gradients of phyllostomid bats across the Neotropic. (a)

1151 Observed entropy. The subplot within shows strong negative correlation between

1152 entropy and temperature seasonality, with highest entropy values associated with lower

1153 temperature seasonality. (b) mean entropy estimated using ABC analysis. Mean values

1154 were computed for simulations with OU's $\alpha$ and $w$ slopes falling within the boundaries

1155 of the $\mathrm{HPD}_{1} 90 \%$ (see fig. 6) computed for the corresponding posterior distribution of

1156 each estimated parameter. (c) Residual values of the cubic model relating estimated (x-

1157 axis) and observed entropy (y-axis) (see subplot within the main plot). 